\title{
DAMA/LIBRA annual modulation and axion quark nugget dark matter model
}

\author{
Ariel Zhitnitsky $\odot$ \\ Department of Physics and Astronomy, University of British Columbia, \\ Vancouver, British Columbia V6T 1Z1, Canada
}

(Received 22 September 2019; accepted 6 April 2020; published 16 April 2020)

\begin{abstract}
The DAMA/LIBRA (DL) experiment shows $9.5 \sigma$ evidence for an annual modulation in the (1-6) keV energy range, strongly suggesting that the observed modulation has a dark matter origin. However, the conventional interpretation in terms of weakly interacting massive particle-nucleon interaction is excluded by other experiments. We propose an alternative source of modulation in the form of neutrons, which have been liberated from surrounding material. Our computations are based on the so-called axion quark nugget (AQN) dark matter model, which was originally invented long ago to explain the similarity between the dark and visible cosmological matter densities, i.e., $\Omega_{\text {dark }} \sim \Omega_{\text {visible }}$. In our proposal, the annual modulation is shown to be generated in the keV energy range, which is consistent with the DL observation in (1-6) keV range. This keV energy scale in our proposal is mostly determined by spectral properties of the neutrinos emitted by the AQN dark matter particles, while the absence of the modulation with energies above $6 \mathrm{keV}$ is explained by a sharp cutoff in the neutrino's energy spectrum at $\sim 15 \mathrm{MeV}$. This proposal can be directly tested by COSINE-100, ANAIS-112, CYGNO, and other similar experiments. It can be also tested by studying the correlations between the signals from these experiments and the signatures from drastically different detectors designed for studies of infrasonic or seismic events using such instruments as distributed acoustic sensing.
\end{abstract}

DOI: 10.1103/PhysRevD.101.083020

\section{INTRODUCTION}

The DAMA/LIBRA (DL) experiment [1-4] claims the observation for an annual modulation in the (1-6) $\mathrm{keV}$ energy range at $9.5 \sigma$ C.L. The C.L. even higher $(12.9 \sigma)$ for the (2-6) $\mathrm{keV}$ energy range when DAMA/NaI and DLphase 1 can be combined with DL-phase 2 results. The measured period $(0.999 \pm 0.001 \mathrm{yr})$ phase $(145 \pm 5)$ strongly indicate a dark matter (DM) origin of the modulation, the phenomenon which was originally suggested in Ref. [5]; see also Ref. [6].

However, the annual modulation observed by DL is excluded by other direct detection experiments if interpreted in terms of the weakly interacting massive particlenuclei interactions. This motivated a number of alternative explanations for the DL signal. In the present work, we argue that the modulation observed by DL is due to the neutrons surrounding the detector. In this respect, our proposal is similar to the previous suggestions [7-10], where the authors argued that the induced neutrons (which

Published by the American Physical Society under the terms of the Creative Commons Attribution 4.0 International license. Further distribution of this work must maintain attribution to the author(s) and the published article's title, journal citation, and DOI. Funded by SCOAP ${ }^{3}$. have been liberated from material surrounding the detector) may be responsible for the observed annual modulation.

Our proposal is drastically different from previous suggestions [7-10] in one crucial aspect: The neutrons in our case are induced by neutrinos emitted by the axion quark nugget (AQN) dark matter particles. Therefore, the annual modulation observed by DL has a truly genuine DM origin, though it is manifested indirectly in our framework through the following chain:

$\mathrm{AQN} \rightarrow($ neutrinos $) \rightarrow$ (surrounding neutrons $) \rightarrow \mathrm{DL}$.

In this framework, the modulation should obviously show a proper period of $1 \mathrm{yr}$ with a proper phase as the neutrinos from Eq. (1) are originated from dark matter nuggets, and the corresponding time variation will be obviously transferred to the modification of the neutron flux, which eventually generates the modulation observed by DL.

One should emphasize that the emission features of the neutrinos emitted by AQNs such as the intensity and spectrum [which ultimately determines the (1-6) $\mathrm{keV}$ energy recoil for the observed DL annual modulation] have been computed in the AQN model long ago for completely different purposes, and we will use exactly the 
same original parameters of the model without any intention to modify them to fit the DL observations.

We overview the basic ideas of the AQN model in Sec. II. One should emphasize that this model is consistent with all available cosmological, astrophysical, satellite, and groundbased constraints, where AQNs could leave a detectable electromagnetic signature. While the model was initially invented to explain the observed relation $\Omega_{\text {dark }} \sim \Omega_{\text {visible }}$, it may also explain a number of other (naively unrelated) phenomena, such as the excess of galactic emission in different frequency bands. The AQN model may also resolve other, naively unrelated astrophysical mysteries, which include, but are not limited to, the so-called "primordial lithium puzzle" [11], the so-called "Solar corona mystery" $[12,13]$, the recent Experiment to Detect the Global Epoch of Reionization Signature (EDGES) observations [14], and unexpected annual modulation in $\mathrm{x}$ rays in the (2-6) $\mathrm{keV}$ energy band observed by the XMM-Newton observatory [15], among many others. These cosmological puzzles could be resolved within the AQN framework with the same set of physical parameters to be used in the present work to explain the annual modulation observed by DL, without fitting or modifications of any of them.

Our main results are formulated in Sec. III, where we estimate the intensity of modulation due to the neutrino flux emitted by dark matter nuggets when they traverse through Earth. This section is separated to four different subsections, Secs. III A, III B, III C, and III D, according to four different elements of the proposal (1). We use precisely the same set of parameters obtained previously for a different purpose, as reviewed in the Appendix A. We further estimate the neutrino-induced neutron's flux in surrounding rocks, which according to the proposal (1) is the source of the observed DL modulation signal.

In Sec. IV, we comment on DL arguments [1-4] suggesting the irrelevance of the induced neutron flux (due to muons and neutrinos). We explicitly show why DL arguments are not applicable for our proposal (1) with its truly genuine DM nature, though manifested indirectly.

In Sec. VA, we comment on a number of experiments which exclude the DL results, while in Sec. V B, we make a few comments on recent results by COSINE-100 [16-18], ANAIS-112 [19], and the recent proposal CYGNO [20], which have been largely motivated by the DL observations. Finally, in Secs. V C and V D, we suggest a few novel tests which could unambiguously support or rule out the proposal (1).

\section{AXION QUARK NUGGET DM MODEL}

In this section, we overview the AQN model. Specifically, in Sec. II A, we list the most important and very generic features of the framework, which do not depend on specific parameters of the model. In Sec. II B, we overview the observational constraints on a single fundamental parameter of this framework, the average baryon charge of the nugget $\langle B\rangle$, which enters all the observables. Finally, in Sec. II C, we highlight a number of other model-dependent properties, such as ionization features of the AQNs, their survival pattern, their annihilation rate, and some other questions which are relevant for the present studies.

\section{A. Generic features of the AQN model}

The idea that dark matter may take the form of composite objects of Standard Model quarks in a novel phase goes back to quark nuggets [21], strangelets [22], and nuclearities [23]; see also the review in Ref. [24] with a large number of references on the original results. In the models [21-24], the presence of a strange quark stabilizes the quark matter at sufficiently high densities, allowing strangelets being formed in the early Universe to remain stable over cosmological timescales.

The AQN model advocated in Ref. [25] is conceptually similar, with the nuggets being composed of a high-density color superconducting (CS) phase. As with other high-mass dark matter candidates [21-24], these objects are "cosmologically dark" not through the weakness of their interactions but due to their small cross section to mass ratio. As a result, the corresponding constraints on this type of dark matter place a lower bound on their mass rather than the coupling constant.

There are several additional elements in the AQN model in comparison with the older well-known and well-studied constructions [21-24].

(i) First, there is an additional stabilization factor provided by the axion domain walls (with a QCD substructure) which are copiously produced during the QCD transition and which help to alleviate a number of the problems inherent in the older models. ${ }^{1}$

(ii) The core of the AQN is in the CS phase, which implies that the two systems (CS and hadronic) can coexist only in the presence of the external pressure which is provided by the axion domain wall. It should be contrasted with original models [21-24] which must be stable at zero external pressure.

(iii) Another crucial additional element in the proposal is that the nuggets could be made of matter as well as antimatter in this framework.

The direct consequence of this feature is that the dark matter density $\Omega_{\text {dark }}$ and the baryonic matter density $\Omega_{\text {visible }}$

\footnotetext{
${ }^{1}$ In particular, in the original proposal the first-order phase transition was the required feature of the construction. However, it is known that the QCD transition is a crossover rather than the first-order phase transition. It should be contrasted with the AQN framework when the first-order phase transition is not required, as the axion domain wall plays the role of the squeezer. Furthermore, it had been argued that the nuggets [21-24] are likely to evaporate on the Hubble timescale even if they were formed. In the AQN framework, the fast evaporation arguments do not apply, because the vacuum ground state energies in the CS and hadronic phases are drastically different.
} 
will automatically assume the same order of magnitude $\Omega_{\text {dark }} \sim \Omega_{\text {visible }}$ without any fine-tunings and irrespectively to any specific details of the model, such as the axion mass $m_{a}$ or size of the nuggets $R$. Precisely this fundamental consequence of the model was the main motivation for its construction.

The presence of a large amount of antimatter in the form of high-density AQNs leads to many observable consequences as a result of possible (but, in general, very rare) annihilation events between antiquarks from AQNs and baryons from the visible Universe. We highlight below the basic results, accomplishments, and constraints of this model.

Let us recapitulate the original motivation for this model: It is commonly assumed that the Universe began in a symmetric state with zero global baryonic charge and later (through some baryon-number-violating process, nonequilibrium dynamics, and $\mathcal{C P}$ violation effects, realizing three famous Sakharov's criteria) evolved into a state with a net positive baryon number.

As an alternative to this scenario, we advocate a model in which "baryogenesis" is actually a charge separation (rather than charge generation) process in which the global baryon number of the Universe remains zero at all times. In this model, the unobserved antibaryons come to comprise the dark matter in the form of dense nuggets of antiquarks and gluons in the CS phase. The result of this "charge separation" process is two populations of AQNs carrying a positive and a negative baryon number. In other words, the AQN may be formed of either matter or antimatter. However, due to the global $\mathcal{C P}$ violating processes associated with $\theta_{0} \neq 0$ during the early formation stage, the number of nuggets and antinuggets will be different. ${ }^{2}$ This difference is always an order of one effect, irrespectively to the parameters of the theory, the axion mass $m_{a}$, or the initial misalignment angle $\theta_{0}$. We refer to the original papers [43-46] devoted to the specific questions related to the nugget's formation, generation of the baryon asymmetry, and survival pattern of the nuggets during the evolution in the early Universe with its unfriendly environment. The only comment we would like to make here is that the disparity between nuggets $\Omega_{N}$ and antinuggets $\Omega_{\bar{N}}$ generated due to $\mathcal{C P}$ violation unambiguously implies that the baryon contribution $\Omega_{B}$ must be the same order of magnitude as $\Omega_{\bar{N}}$ and $\Omega_{N}$, because all these contributions are proportional to one and the same fundamental dimensional parameter $\Lambda_{\mathrm{QCD}}$. If these processes are not fundamentally related, the two components $\Omega_{\text {dark }}$ and $\Omega_{\text {visible }}$ could easily assume drastically different scales. This

\footnotetext{
${ }^{2}$ This source of strong $\mathcal{C P}$ violation is no longer available at the present epoch as a result of the dynamics of the axion, which remains the most compelling resolution of the strong $\mathcal{C P}$ problem; see original papers on Peccei-Quinn symmetry [26], WeinbergWilczek axion [27,28], Kim-Shifman-Vainshtein-Zakharov invisible axion [29,30], and Dine-Fischler-Srednicki-Zhitnitsky invisible axion [31,32] models. See also recent reviews [33-42].
}

represents a precise mechanism of how the "baryogenesis" can be replaced by the charge separation processes in the AQN framework.

The remaining antibaryons in the early Universe plasma then annihilate away, leaving only the baryons whose antimatter counterparts are bound in the excess of antiquark nuggets and are, thus, unavailable for fast annihilation. All asymmetry effects are of the order of one, which eventually results in similarities for all components, visible and dark, i.e.,

$$
\Omega_{\mathrm{dark}} \sim \Omega_{\mathrm{visible}}, \quad \Omega_{\mathrm{dark}} \approx\left[\Omega_{N}+\Omega_{\bar{N}}\right],
$$

as they are both proportional to the same fundamental $\Lambda_{\mathrm{QCD}}$ scale, and they both are originated at the same QCD epoch. In particular, the observed matter to dark matter ratio $\Omega_{\text {dark }} \simeq$ $5 \cdot \Omega_{\text {visible }}$ corresponds to a scenario in which the number of antinuggets is larger than the number of nuggets by a factor of $\left(\Omega_{\bar{N}} / \Omega_{N}\right) \sim 3 / 2$ at the end of nugget formation. It is important to emphasize that the AQN mechanism is not sensitive to the axion mass $m_{a}$ and it is capable to saturate (2) itself without any other additional contributors. It should be contrasted with conventional axion production mechanisms when the corresponding contribution scales as $\Omega_{\text {axion }} \sim m_{a}^{-7 / 6}$. This scaling unambiguously implies that the axion mass must be fine-tuned $m_{a} \simeq 10^{-5} \mathrm{eV}$ to saturate the DM density today, while a larger axion mass will contribute very little to $\Omega_{\text {dark }}$. The relative role between the direct axion contribution $\Omega_{\text {axion }}$ and the AQN contribution to $\Omega_{\text {dark }}$ as a function of mass $m_{a}$ has been evaluated in Ref. [45]; see Fig. 5 in that paper.

Unlike conventional dark matter candidates, such as weakly interacting massive particles (WIMPs), the dark matter or antimatter nuggets are strongly interacting and macroscopically large. However, they do not contradict any of the many known observational constraints on dark matter or antimatter due to the following main reasons [47].

(i) They are absolutely stable configurations on the cosmological scale, as the pressure due to the axion domain wall (with the QCD substructure) is equilibrated by the Fermi pressure. Furthermore, it has been shown that the AQNs survive the unfriendly environment of the early Universe, before and after the big bang nucleosynthesis (BBN) epoch [46]. The majority of the AQNs also survive such violent events as galaxy formation and star formation.

(ii) They carry a huge (anti)baryon charge $|B| \gtrsim 10^{25}$ and so have an extremely tiny number density.

(iii) The nuggets have nuclear densities, so their effective cross section $\sigma \sim R^{2}$ is small relative to its mass, $\sigma / M \sim 10^{-10} \mathrm{~cm}^{2} / \mathrm{g}$. This key ratio is well below the typical astrophysical and cosmological limits which are on the order of $\sigma / M<1 \mathrm{~cm}^{2} / \mathrm{g}$.

(iv) They have a large binding energy such that the baryon charge locked in the nuggets is not available to participate in $\mathrm{BBN}$ at $T \sim 0.1 \mathrm{MeV}$, and the basic BBN picture holds, with possible small corrections 
of the order of $\sim 10^{-10}$ which may, in fact, resolve the primordial lithium puzzle [11].

(v) The nuggets completely decouple from photons as a result of the small $\sigma / M \sim B^{-1 / 3} \ll 1$ ratio, such that the conventional picture for structure formation holds.

(vi) The nuggets do not modify conventional cosmic microwave background analysis, with possible small corrections which, in fact, may resolve a tension [14] between the standard prediction and EDGES observation (stronger than anticipated $21 \mathrm{~cm}$ absorption features).

To reiterate, the weakness of the visible-dark matter interaction is achieved in this model due to the small geometrical factor $\sigma / M \sim B^{-1 / 3}$ rather than due to a weak coupling of a new fundamental field to Standard Model particles. In other words, this small effective interaction $\sim \sigma / M \sim B^{-1 / 3}$ replaces a conventional requirement of sufficiently weak interactions of the visible matter with WIMPs.

\section{B. Mass distribution constraints}

One should emphasize that the AQN construction does not specify the size of the nuggets. In general, for a given axion mass $m_{a}$ there is always a range of $B$ when a stable solution exists [25]. The average size of the nugget within this stability range scales as $R \propto m_{a}^{-1}$, while the baryon charge of the AQN itself scales as $\langle B\rangle \propto R^{3} \propto m_{a}^{-3}$. In the AQN framework we treat $\langle B\rangle$ as a fundamental parameter to be constrained observationally. It is clear that larger $\langle B\rangle$ values produce weaker observational signals as $\sigma / M \sim B^{-1 / 3}$. Furthermore, any such consequences assume the largest values where the densities of both visible and dark matter are sufficiently high such as in the core of the Galaxy, the early Universe, or the stars and planets. In other words, the nuggets behave like conventional cold dark matter in environments where the density of the visible matter is small, while they become interacting and radiating objects (i.e., effectively become visible matter) if they enter an environment of sufficiently large density.

As mentioned above, the flux of AQNs on Earth's surface is scaled by a factor of $B^{-1}$ and is thus suppressed for large nuggets; see Eq. (8) below. For this reason, the experiments most relevant to AQN detection are not the conventional high-sensitivity dark matter searches but detectors with the largest possible search area. For example, it has been proposed [48] that large-scale cosmic ray detectors such as the Auger observatory of Telescope Array may be sensitive to the flux of AQNs in an interesting mass range. An obvious challenging problem with such studies is that the conventional cosmic ray detectors are designed to analyze the time delays measured in microns, as cosmic rays are assumed to be moving with the speed of light, while AQNs move with nonrelativistic velocity $v_{\mathrm{AQN}} \simeq 10^{-3} c$. It obviously requires the latency time to be measured in the millisecond range in order to study the correlated signals from AQNs. The modern cosmic ray detectors are not designed to analyze such long time correlations, and normally such signals would be rejected as a background.

The strongest direct detection limit is set by the IceCube Observatory's nondetection of a nonrelativistic magnetic monopole [49]. While the magnetic monopoles and the AQNs interact with material of the detector in very different ways, in both cases the interaction leads to electromagnetic and hadronic cascades along the trajectory of the AQN (or magnetic monopole) which must be observed by the detector if such an event occurs. The nonobservation of any such cascades puts the following limit on the flux of heavy nonrelativistic particles passing through the detector:

$\langle B\rangle>3 \times 10^{24} \quad$ [direct (non)detection constraint],

where we assume $100 \%$ efficiency of the observation of the AQNs passing through IceCube Observatory; see Appendix A in Ref. [50].

Similar limits are also derivable from the Antarctic Impulsive Transient Antenna (ANITA) [51], though this result depends on the details of radio band emissivity of the AQN. In the same work, the author also derives the constraint arising from a potential contribution of the $\mathrm{AQN}$ annihilation events to Earth's energy budget which requires $|B|>2.6 \times$ $10^{24}$ [51], which is also consistent with Eq. (3). There is also a constraint on the flux of heavy dark matter with mass $M<$ $55 \mathrm{~g}$ based on the nondetection of etching tracks in ancient mica [52]. It slightly touches the lower bound of the allowed window (3) but does not strongly constraint the entire window (5), because the dominant portion of the AQNs lies well above its lower limit (3) assuming the mass distribution (6) as discussed below.

The authors of Ref. [53] use the Apollo data to constraint the abundance of quark nuggets in the region of $10 \mathrm{~kg}$ to one ton. It has been argued that the contribution of such very heavy nuggets must be at least an order of magnitude less than would saturate the dark matter in the Solar neighborhood [53]. Assuming that the AQNs saturate the dark matter, the constraint [53] can be reinterpreted that at least $90 \%$ of the AQNs must have masses below $10 \mathrm{~kg}$. This constraint can be approximately expressed in terms of the baryon charge as follows:

$$
\langle B\rangle \lesssim 10^{28} \quad \text { [Apollo constraint]. }
$$

Therefore, indirect observational constraints (3) and (4) suggest that, if the AQNs exist and saturate the dark matter density today, the dominant portion of them must reside in the window:

$3 \times 10^{24} \lesssim\langle B\rangle \lesssim 10^{28} \quad$ [constraints from observations].

Completely different and independent observations also suggest that the galactic spectrum contains several excesses 
of diffuse emission, the origin of which is not well established and remains to be debated. The best known example is the strong galactic $511 \mathrm{keV}$ line. If the nuggets have a baryon number in the $\langle B\rangle \sim 10^{25}$ range, they could offer a potential explanation for several of these diffuse components. It is a very nontrivial consistency check that the required $\langle B\rangle$ to explain these excesses of the galactic diffuse emission belongs to the same mass range as reviewed above. For further details, see the original works [54-59] with explicit computations of the galactic radiation excesses for various frequencies, including excesses of the diffuse $\mathrm{x}$ and $\gamma$ rays. In all these cases, photon emission originates from the outer layer of the nuggets known as the electrosphere, and all intensities in different frequency bands are expressed in terms of a single parameter $\langle B\rangle$ such that all relative intensities are unambiguously fixed, because they are determined by the Standard Model physics.

Yet another AQN-related effect might be intimately linked to the so-called "Solar corona heating mystery." The renowned (since 1939) puzzle is that the corona has a temperature $T \simeq 10^{6} \mathrm{~K}$, which is 100 times hotter than the surface temperature of the Sun, and conventional astrophysical sources fail to explain the extreme UV (EUV) and soft x-ray radiation from the corona $2000 \mathrm{~km}$ above the photosphere. Our comment here is that this puzzle might find its natural resolution within the AQN framework as recently argued in Refs. [12,13,60].

In this scenario, the AQNs composed of antiquarks fully annihilate within the so-called transition region, providing a total annihilation energy which is very close to the observed EUV luminosity of $10^{27} \mathrm{erg} / \mathrm{s}$. The EUV emission is assumed to be powered by impulsive heating events known as nanoflares conjectured by Parker long ago. The physical origin of the nanoflares remains unknown. If our identification of these nanoflares with the AQN annihilating events is correct, we may extract the baryon charge distribution $d N / d B$ for the AQNs, because the energy distribution $d N / d E$ of the nanoflares has been previously modeled by the solar physics people to fit the observations, i.e.,

$$
d N \propto E^{-\alpha} d E \propto B^{-\alpha} d B
$$

where slop parameter $\alpha$ slightly varies for different solar models. ${ }^{3}$ It is a highly nontrivial consistency check that the typical nanoflare energy range $E_{\text {nano }} \simeq\left(10^{22}-6 \times 10^{25}\right) \mathrm{erg}$

\footnotetext{
${ }^{3}$ One should comment here that the algebraic behavior for the distribution (6) is a very generic feature of the percolation theory within the AQN framework as recently argued in Ref. [46]. However, a numerical estimate of parameter $\alpha$ from the theoretical side requires a deep understanding of the QCD phase transition dynamics at $\theta \neq 0$, when the axion domain walls are formed. This knowledge is not likely to be available any time soon, as the QCD lattice simulations cannot study a system with $\theta \neq 0$, which represents a well-known sign problem in the lattice community.
}

corresponds (within the AQN framework) to the baryon charge window $3 \times 10^{24} \lesssim B \lesssim 2 \times 10^{28}$, which strongly overlaps with all presently available constraints (5) on the AQN sizes as reviewed above. Precisely this highly nontrivial consistency check on size distribution along with the (essentially model-independent) computation of the total luminosity of the EUV radiation $\sim 10^{27} \mathrm{erg} / \mathrm{s}$ from the Solar corona, which is consistent with observations, gives us a strong confidence for the plausibility of the identification (6) between the nanoflares conjectured by Parker long ago and the AQN annihilation events.

Encouraged by these consistency checks, we adopted the AQN size distribution (6) with parameter $\alpha$ being extracted from the heating corona studies for all our subsequent Monte Carlo simulations including the computations of the AQN flux on Earth as given by (8) to be discussed in great detail in the next section.

\section{A few additional comments}

Here, we make a few additional comments on basic features of the AQNs which are important for the understanding of this framework, in general, and for specific estimates (1) relevant for DAMA/LIBRA signal, in particular.

We start this overview with a mention of the electric charge which AQNs may carry while propagating in a medium. It is normally assumed that all types of nuggets including the old version models [21-24] are neutral at zero temperature $T=0$, because the electrosphere made of leptons will be always formed even when the quark nuggets themselves are electrically charged (for example, due to the differences in the quark's masses). However, the neutrality will be lost due to the ionization at $T \neq 0$, in which case the nuggets will esquire the positive charge due to the ionized electrons, while the antimatter nuggets will esquire the negative electric charge due to the ionized positrons. The corresponding charge $Q$ for AQNs can be estimated as follows [11,12]:

$$
Q \simeq 4 \pi R^{2} \int_{z_{0}}^{\infty} n(z) d z \sim \frac{4 \pi R^{2}}{2 \pi \alpha} \cdot\left(T \sqrt{2 m_{e} T}\right),
$$

where $n(z)$ is the density of the positrons in the electrosphere which has been computed in the mean field approximation. In this estimate, it is assumed that the positrons with $p^{2} /\left(2 m_{e}\right)<T$ will be stripped off the electrosphere as a result of high temperature $T$. These loosely bound positrons are localized mostly at outer regions of the electrosphere at distances $z>z_{0}=\left(2 m_{e} T\right)^{-1 / 2}$, which motivates our cutoff in estimate (7). Numerically, $Q \sim 10^{8}$ represents very tiny portion $Q / B$ in comparison with the baryon charge $B \sim 10^{25}$ even for relatively high temperature $T \simeq 100 \mathrm{eV}$ in the Solar corona. These objects behave in all respects as neutral objects (for example, the cosmic magnetic field does not 
affect the AQN's trajectory) because $Q / M \ll e / m_{p}$. Nevertheless, a nonvanishing charge $Q$ may play a very important role in some circumstances, such as the propagation of AQNs in highly ionized plasma in the Solar corona $[12,13,60]$. The nonvanishing charge $Q$ may also suppress the primordial lithium abundance at $T \simeq 20 \mathrm{keV}$ due to the strong attraction between the negatively charged AQNs and positively charged lithium nuclei [11].

Our next comment is related to the survival pattern of the AQNs. Comprehensive studies on this matter can be found in the original work [46]. The only comment we would like to make here is that the dominant portion of the AQNs will survive the evolution of the Universe. However, a very small portion of the AQNs which are gravitationally captured by stars and planets may drastically decrease their baryon charges and may even experience complete annihilation. ${ }^{4}$ The typical size $L$ of the region of the medium with density $\rho$ where the complete annihilation occurs is estimated as $\sigma \rho L \simeq B m_{p}$, where $\sigma$ is an effective cross section which could be much larger than naive $\pi R^{2}$ due to the long-range Coulomb interaction when $Q \neq 0$ according to Eq. (7). This effect plays a very important role in the Solar corona $[12,13,60]$. All nuggets which are gravitationally captured by the Sun will be completely annihilated.

One may wonder what happens to the axions from AQNs which are now liberated and become propagating axions with average energy $\langle E\rangle \simeq 1.3 m_{a}$. A small portion of these axions will be converted to photons in the background of the Solar magnetic field and, in principle, can be observed on Earth. The effect is very small, though, even if one takes into account the resonance condition due to the plasma effects in the Solar corona [61].

In the case of the AQN traversing Earth's interior, only some small portion of the baryon charge will be annihilated. The full-scale Monte Carlo simulations suggest that on average approximately (10-20)\% of the total baryon charge will be lost as a result of traversing of the AQNs through Earth's interior; see the column for $\Delta B / B$ in Table III in Ref. [50]. The average amount of the lost baryon charge depends, of course, on the size distribution and parameter $\alpha$ defined by Eq. (6). To avoid confusion, let us emphasize one more time that all these AQNs which get

\footnotetext{
${ }^{4}$ The nuggets made of matter can be stopped in a dense environment such as neutron stars, on scales of the order of $L^{\text {stop }}$, when the number of hits is of the order of $B$, i.e., $\pi R^{2} L^{\text {stop }}\langle\rho\rangle \sim B m_{p}$. However, in contrast with conventional nugget's models [21-24], the AQNs will not turn an entire star into a new phase, because the CS phase in the AQN is supported by external pressure due to the axion domain wall, while the original nuggets [21-24] are assumed to be stable objects at zero external pressure. The phenomenon of collecting the matter nuggets in the cores of stars or planets might be of interest by itself, but it is not the topic of the present work devoted to antimatter AQNs capable of producing neutrinos as a result of annihilation processes.
}

completely annihilated or lost a finite portion of their baryon charges represent a very tiny portion of all AQNs during the entire evolution of the Universe as estimated in Ref. [46].

A final comment we would like to make in this subsection is related to spallation, which represents a very common process when heavy nuclei lose their baryon charge as a result of interaction with a medium. In contrast with conventional nuclei, spallation cannot play any essential role in the AQN survival pattern due to several reasons. First of all, the gap $\Delta$ in the CS phase is typically in the $100 \mathrm{MeV}$ range, in contrast with conventional nuclear physics where the binding energy normally is in the few $\mathrm{MeV}$ range. The most important distinct feature, however, is as follows. When a large amount of energy is injected into a heavy nucleus, spallation takes place and a large number of neutrons may be liberated, leading to the decreasing of the baryon charge of the heavy original nucleus. Such a process cannot occur with AQNs, because all particles which get excited due to the energy injection in the CS phase are the colored objects. Therefore, these elementary excitations cannot enter the hadronic vacuum where the normal baryons live and must stay inside of the $A Q N$. Therefore, the AQNs do not suffer from spallation processes as heavy nuclei do. This is a direct manifestation of the same feature of the AQN construction already mentioned in footnote 1 , which states that the confined hadronic and CS phases have different vacua. The same feature precludes transformation of the entire star or planet into a new phase if the AQNs made of matter stop in the deep interior of the star or planet; see also footnote 4 .

\section{DL MODULATION BY AQNS}

This section is separated into four different subsections, Secs. III A, III B, III C, and III D, according to four different elements of the proposal (1).

\section{A. AQN flux on Earth}

We start our task with the first element from proposal (1) by estimating the AQN hit rate per unit area on Earth's surface assuming that $\rho_{\mathrm{DM}}$ is entirely saturated by the nuggets. The relevant rate has been studied previously in Ref. [50] for a different problem of computing the axion flux produced by the AQNs. Now we estimate the AQN hitting rate assuming conventional dark matter density $\rho_{\text {DM }} \simeq 0.3 \mathrm{GeV} \mathrm{cm}^{-3}$ surrounding Earth. Assuming the conventional halo model, one arrives to the following result [50]:

$$
\frac{\langle\dot{N}\rangle}{4 \pi R_{\oplus}^{2}} \simeq \frac{4 \times 10^{-2}}{\mathrm{~km}^{2} \mathrm{yr}}\left(\frac{\rho_{\mathrm{DM}}}{0.3 \frac{\mathrm{GeV}}{\mathrm{cm}^{3}}}\right)\left(\frac{\left\langle v_{\mathrm{AQN}}\right\rangle}{220 \frac{\mathrm{km}}{\mathrm{s}}}\right)\left(\frac{10^{25}}{\langle B\rangle}\right) .
$$

The averaging over all types of $\mathrm{AQN}$ trajectories with different masses $M_{N} \simeq m_{p}|B|$, with different incident 
angles, different initial velocities, and different size distributions does not modify much this estimate. The result (8) suggests that the AQNs hit Earth's surface with a frequency approximately once a day per $100^{2} \mathrm{~km}^{2}$ area. The hitting rate for large size objects is suppressed by the distribution function $f(B) \propto B^{-\alpha}$ as given by Eq. (6).

The estimate (8) explicitly shows that conventional DM detectors are too small in size to detect AQNs directly, as the corresponding flux is many orders of magnitude smaller than the one due to the conventional WIMPs. However, some modern cosmic ray detectors, such as Pierre Auger observatory, in principle, are capable to study small flux of the order of Eq. (8) as suggested in Ref. [48]. One should also mention that a smaller size IceCube detector imposes a direct constraint on the average baryon charge of the nugget $\langle B\rangle \geq 3 \times 10^{24}$; see Appendix A in Ref. [50].

From Eq. (8), one can derive the total hit rate for the entire Earth's surface, which is given by [50]

$$
\langle\dot{N}\rangle \simeq 0.67 \mathrm{~s}^{-1}\left(\frac{\rho_{\mathrm{DM}}}{0.3 \frac{\mathrm{GeV}}{\mathrm{cm}^{3}}}\right)\left(\frac{\left\langle v_{\mathrm{AQN}}\right\rangle}{220 \frac{\mathrm{km}}{\mathrm{s}}}\right)\left(\frac{10^{25}}{\langle B\rangle}\right) .
$$

After the nugget hits the surface, it continues to propagate by annihilating the material along its path. The trajectory of the AQN is a straight line, as only a small portion of the momentum (and the baryon charge) will be lost in this journey. The energy produced due to the annihilation events will be isotropically dissipating (in the rest frame of the nugget) along the propagation.

The rate (9) includes all types of the AQN's trajectories inside Earth's interior: the trajectories when AQNs hit the surface with an incident angle close to $0^{\circ}$ (in which case the AQN crosses Earth's core and exits from the opposite site of Earth) as well as the trajectories when AQNs just touch the surface with an incident angle close to $90^{\circ}$ (when AQNs leave the system without many annihilation events deep underground). The result of summation over all these trajectories can be expressed in terms of the average mass (energy) loss $\left\langle\Delta m_{\mathrm{AQN}}\right\rangle$ per AQN. The same information can also be expressed in terms of the average baryon charge loss per nugget $\langle\Delta B\rangle$, as these two are directly related: $\left\langle\Delta m_{\mathrm{AQN}}\right\rangle \approx m_{p}\langle\Delta B\rangle$. The corresponding Monte Carlo simulations with estimates for $\left\langle\Delta m_{\mathrm{AQN}}\right\rangle$ have been carried out in Ref. [50]; see Table III in that paper. This information will be very important for our analysis in Sec. III B, as it provides a normalization for the total neutrino flux being emitted by the AQNs when they traverse Earth's interior.

\section{B. Neutrino production from AQNs}

The second element of the proposal (1) requires the estimation of the neutrino intensity due to the AQN annihilation processes. Before we proceed with the corresponding estimates, we want to make a short detour related to the axion production due to the same AQN annihilation events. We also need to know the basic features of the neutrino spectrum emitted by AQNs. The lessons from that studies can be used for estimations of the neutrino flux from AQNs, which is the main subject of this subsection.

\section{Detour on the axion production}

It has been noticed in Ref. [62] that the large number of axions will be produced, because the axion domain wall ${ }^{5}$ will start to shrink during the AQN annihilation events and emit the propagating axions which can be observed. The corresponding spectrum has been computed in Ref. [61], where it has been shown that the emitted axions will have typical velocities $\left\langle v_{a}\right\rangle \simeq 0.6 c$ in contrast with conventional galactic axions characterized by small velocities $\sim 10^{-3} \mathrm{c}$ such that these two different production mechanisms can be easily discriminated.

The average number of the emitted axions $\left\langle N_{a}\right\rangle$ as a result of the AQN annihilation events in Earth's interior can be estimated as follows [63]:

$$
\left\langle N_{a}\right\rangle \approx \kappa_{a} \frac{\left\langle\Delta m_{\mathrm{AQN}}\right\rangle}{\left\langle E_{a}\right\rangle}
$$

where coefficient $\kappa_{a}$ determines the relative amount of annihilating energy (per unit baryon charge) transferred to the axion production. The computation of the coefficient $\kappa_{a} \simeq 1 / 3$ as well as $\left\langle E_{a}\right\rangle \simeq 1.3 m_{a}$ is a straightforward exercise [61], as it represents a conventional quantum field theory problem for a weakly interacting axion field.

The energy flux of the axions (being averaged over all emission angles and summed over all trajectories) measured on Earth's surface is estimated as [63]

$$
\frac{d E_{a}}{d t d A} \simeq \kappa_{a}\left\langle\frac{v_{a}}{c}\right\rangle\langle\dot{N}\rangle \frac{\left\langle\Delta m_{\mathrm{AQN}} c^{2}\right\rangle}{2 \pi R_{\oplus}^{2}},
$$

which has a proper dimensionality $\left[\mathrm{GeV} \cdot \mathrm{cm}^{-2} \cdot \mathrm{s}^{-1}\right]$ for the energy flux. The axion flux for this mechanism is estimated as

$$
\frac{d N_{a}}{d t d A} \simeq \kappa_{a}\left\langle\frac{v_{a}}{c}\right\rangle\langle\dot{N}\rangle \frac{\left\langle\Delta m_{\mathrm{AQN}} c^{2}\right\rangle}{\left\langle E_{a}\right\rangle 2 \pi R_{\oplus}^{2}},
$$

which has a proper dimensionality $\left[\mathrm{cm}^{-2} \cdot \mathrm{s}^{-1}\right]$ for the axion flux.

We are now ready to estimate the neutrino emission from the AQNs, which is the main topic of this subsection. One could follow the same logic of computations as highlighted

\footnotetext{
${ }^{5}$ As mentioned in Sec. II, the axion domain wall plays the important role of a squeezer stabilizing the nugget. This energy has been accumulated and stored during the QCD epoch at the moment of formation. The corresponding energy accounts for a considerable portion of the nugget's total energy, which is parametrized by $\kappa_{a} \approx 1 / 3$; see below Eq. (10).
} 
above, with the only difference is that instead of emission of the axions one should study the neutrinos, which are similar to axions, as they can easily propagate through Earth's entire interior. This is because the relevant cross section with surrounding material is very small in both cases, and formula (11) can be applied for estimation of the neutrino flux on Earth's surface with corresponding replacements $\kappa_{a} \rightarrow \kappa_{\nu}$ and $E_{a} \rightarrow E_{\nu}$, while factor $\langle\dot{N}\rangle$ in Eq. (12), of course, remains the same.

\section{On neutrino spectrum in the CS phase}

If we had a conventional hadronic phase inside the nuggets, the corresponding computations of neutrino emission would be a very simple exercise. Indeed, we know a typical yield of the pseudo-Nambu-Goldstone (NG) bosons per annihilation event of a single baryon charge (such as $\bar{p} p$ ). We also know a typical decay pattern for all NG bosons such as $K \rightarrow \mu \nu$ and $\pi \rightarrow \mu \nu$ with consequent $\mu$ decays. We also know with very high precision the branching ratios for the nonleptonic decays of the NG bosons such as $K \rightarrow 3 \pi$ and $\eta \rightarrow 3 \pi$ with consequent decays to neutrinos. It would allow us to compute the total number of neutrinos per single annihilation event. This would also allow us to compute the energy spectrum of neutrinos ${ }^{6}$ emitted by the AQNs.

Unfortunately, we do not have this luxury of knowing all these key features in the CS phase. Therefore, we cannot predict the spectrum of neutrinos emitted by AQNs. The only solid and robust information which is available today is the typical scale of the lightest NG mass in the CS phase, which is normally estimated in the (20-30) MeV range. This scale for NG masses can be translated to an estimation for a typical neutrino's energy scale in the AQN framework. Assuming that one-half of the lightest NG mass goes to the neutrino's energy, we expect that $\left\langle E_{\nu}\right\rangle \lesssim 15 \mathrm{MeV}$.

While a typical energy scale for the NG mass and the corresponding neutrino's energies in the AQN framework is established with a reasonable accuracy because it is based on the well-established theory of CS phases $[66,67]$, the computation of the corresponding neutrino spectrum is a much harder problem. The basic problem is, of course, the

\footnotetext{
${ }^{6}$ This is precisely the set of assumptions adopted by Ref. [64], where the authors claimed that dark matter in the form of AQNs cannot account for more than $20 \%$ of the dark matter density. This claim was based on the assumption that the annihilation events follow the conventional (for a confined phase) pattern, in which case a large number of neutrinos will be produced in the (20-50) $\mathrm{MeV}$ range, where the sensitivity of underground neutrino detectors such as Super-Kamiokande have their highest signal-to-noise ratio. The basic claim of Ref. [65] is that annihilation processes involving an antiquark nugget in the CS phase proceed in a drastically different way than assumed in Ref. [64] when the lightest pseudo-Nambu-Goldstone meson has a mass in the (20-30) MeV range. As a result of this crucial difference, the neutrino's energies will be in the $15 \mathrm{MeV}$ range, well below the present day constraints.
}

lack of knowledge of the CS phase (in contrast with the confined phase, where all NG masses and relevant branching ratios are measured with very high accuracy). An additional uncertainty also comes from the lack of understanding of the fermion excitations of the CS phases which may be, in fact, the dominant contributors to the neutrino fluxes. This is because the NG bosons which are produced as a result of annihilation events in the CS phase cannot leave the system and consequently decay to emit neutrinos, as they must stay inside the nuggets. ${ }^{7}$ It should be contrasted with the hadronic phase when pions and kaons (produced as a result of $p \bar{p}$ annihilation) decay to muons and neutrinos in a vacuum. Therefore, the NG bosons in CS phases are likely to be absorbed by fermion excitations (if kinematically allowed), which consequently decay to neutrinos.

Indeed, in unpaired quark matter, neutrino emissivity is dominated by the direct Urca processes ${ }^{8}$ such as $d \rightarrow u+e^{-}+\bar{\nu}_{e}$. In the case of antinuggets, it should be replaced by antiquarks with the emission of neutrino $\nu_{e}$ and positron $e^{+}$with the energy determined by the energy of the fermion excitation, which itself assumes the energy of the order of the NG mass; see Appendix A 3 with more comments on this matter.

If this process indeed becomes the dominant mechanism of the neutrino emission from AQNs, then one should expect that the number of neutrinos per single event of annihilation should greatly exceed the number of antineutrinos per single event of annihilation, which we assume to be the case. Formally, this case can be expressed as follows:

$$
\begin{aligned}
\left\langle N_{\nu}\right\rangle & \approx \kappa_{\nu}\left[\frac{\left\langle\Delta m_{\mathrm{AQN}} c^{2}\right\rangle}{m_{p} c^{2}}\right], \quad\left\langle N_{\bar{\nu}}\right\rangle \approx \kappa_{\bar{\nu}}\left[\frac{\left\langle\Delta m_{\mathrm{AQN}} c^{2}\right\rangle}{m_{p} c^{2}}\right], \\
\kappa_{\nu} & \gg \kappa_{\bar{\nu}}, \quad E_{\nu} \lesssim 15 \mathrm{MeV}, \quad \kappa_{\nu} \gtrsim 1,
\end{aligned}
$$

where the coefficients $\kappa_{\nu}$ and $\kappa_{\bar{\nu}}$ describe the number of neutrinos and antineutrinos, respectively, produced per single annihilation event, similar to parameter $\kappa_{a} \simeq 1 / 3$ entering expression (10) and describing the axion emission due to the AQN annihilation events.

We conclude this subsection with the following generic comment. Our system is the strongly coupled gauge theory, the QCD. It should be contrasted with conventional weakly coupled gauge theories when all computations are under complete theoretical control. In our system, it is very hard to predict realistic spectra and intensities for neutrino and

\footnotetext{
${ }^{7}$ This is because all excitations in the CS phase are color objects and cannot propagate in a hadronic vacuum.

${ }^{8}$ Another direct Urca process $e^{-}+u \rightarrow d+\nu_{e}$ which for antinuggets would correspond to emission of the antineutrino is likely to be strongly suppressed, as it requires the presence of positrons with sufficiently high energy above the Fermi surface. For a low temperature, the corresponding density for positron excitations is exponentially small $\sim \exp \left(-E_{e^{+}} / T\right)$. This argument suggests that $\kappa_{\nu} \gg \kappa_{\bar{\nu}}$ as Eq. (13) states.
} 
antineutrino fluxes in the $15 \mathrm{MeV}$ energy range due to a variety of possible phases, high sensitivity to the parameters, and a large number of possible decay channels producing neutrinos and antineutrons, as we discussed above. Such an analysis could be coined as "the nuclear physics of CS phases." The complexity and uncertainties of such studies (though it is entirely based on the Standard Model physics) are the main reasons to introduce phenomenological parameters $\kappa_{\nu}$ and $\kappa_{\bar{\nu}}$ in Eq. (13), which will be treated in what follows as unknown parameters and can be constrained only by experiment. However, the basic scale of the problem is fixed by the lowest NG mass in the CS phase with a reasonable accuracy, and it is given by Eq. (13), i.e., $\left\langle E_{\nu}\right\rangle \lesssim 15 \mathrm{MeV}$. Precisely this basic neutrino energy scale determines the maximum recoil energy around $6 \mathrm{keV}$ in the DL signal.

\section{Neutrino flux from $A Q N s$}

In what follows, we need the expression for the neutrino flux similar to our formula for the axion flux (12):

$$
\frac{d N_{\nu}}{d t d A} \simeq \kappa_{\nu}\langle\dot{N}\rangle \frac{\left\langle\Delta m_{\mathrm{AQN}}\right\rangle}{2 \pi R_{\oplus}^{2} m_{p}} .
$$

A similar expression is also valid for antineutrinos: One should replace $\kappa_{\nu} \rightarrow \kappa_{\bar{\nu}}$ and $d N_{\nu} \rightarrow d N_{\bar{\nu}}$ in Eq. (14). Now we are ready for the numerical estimates. We use $\left\langle\Delta m_{\mathrm{AQN}}\right\rangle$ and $\langle\dot{N}\rangle$ from Sec. III A to arrive to the following estimate for the neutrino flux in terms of unknown parameter $\kappa_{\nu}$ :

$$
\frac{d N_{\nu}}{d t d A} \simeq 0.6 \times 10^{6} \cdot \kappa_{\nu} \cdot\left(\frac{\langle\Delta B\rangle}{\langle B\rangle}\right) \frac{1}{\mathrm{~cm}^{2} \cdot \mathrm{s}}
$$

and a similar expression for antineutrinos obtained from Eq. (15) by replacing $\kappa_{\nu} \rightarrow \kappa_{\bar{\nu}}$ and $d N_{\nu} \rightarrow d N_{\bar{\nu}}$. In formula (15), the dimensionless ratio $\langle\Delta B\rangle /\langle B\rangle$ counts the relative portion of baryon charge being annihilated in the interior while AQNs traverse Earth. This parameter depends on the nugget's size distribution as reviewed in Sec. II B. Numerically, it is close to $10 \%$ for models with large average charge $\langle B\rangle \simeq 10^{26}$ and around $30 \%$ for models with smaller average charge $\langle B\rangle \simeq 10^{25}$ [50]. In what follows, to simplify things we want to ignore all these numerical factors and represent the total neutrino and antineutrino fluxes produced by the AQN mechanism over the entire energy range $0 \lesssim E_{\nu, \bar{\nu}} \lesssim 15 \mathrm{MeV}$ as follows:

$\frac{d N_{\nu}}{d t d A} \sim 10^{5} \cdot \kappa_{\nu} \frac{1}{\mathrm{~cm}^{2} \cdot \mathrm{s}}, \quad \frac{d N_{\bar{\nu}}}{d t d A} \sim 10^{5} \cdot \kappa_{\bar{\nu}} \frac{1}{\mathrm{~cm}^{2} \cdot \mathrm{s}}$.

It is instructive to quote a few known constraints on neutrino and antineutrino fluxes in this energy band $E_{\nu, \bar{\nu}} \sim$ $15 \mathrm{MeV}$ in order to compare them with the fluxes produced by the AQN mechanism as expressed by Eq. (16). The largest flux relevant for this energy band comes from solar ${ }^{8} B$, which is around $\Phi_{\nu_{e}} \simeq 5 \times 10^{6}\left(\mathrm{~cm}^{-2} \mathrm{~s}^{-1}\right)$, while the solar "hep" component is close to $\Phi_{\nu_{e}} \simeq 8 \times 10^{3}\left(\mathrm{~cm}^{-2} \mathrm{~s}^{-1}\right)$ [68]. The atmospheric and diffuse supernova neutrino backgrounds are at least 2 orders of magnitude smaller than the hep component and can be safely ignored in our discussions.

One should also mention the Super-Kamiokande stringent constraint on antineutrino flux $\Phi_{\bar{\nu}_{e}}<(1.4-1.9) \mathrm{cm}^{-2} \mathrm{~s}^{-1}$ at large energies $E>19.3 \mathrm{MeV}$ [69] and constraint $\Phi_{\bar{\nu}_{e}}<$ $4 \times 10^{4} \mathrm{~cm}^{-2} \mathrm{~s}^{-1}$ at smaller energies $8 \mathrm{MeV}<E<$ $20 \mathrm{MeV}$ [70]. The KamLand Collaboration [71] reports a model-independent upper limit on the antineutrino flux $\Phi_{\bar{\nu}_{e}}<10^{2} \mathrm{~cm}^{-2} \mathrm{~s}^{-1}$ for every energy bin between 8.3 and 11.3 MeV which becomes even stronger for higher energies.

To conclude this subsection, we would like to emphasize that the neutrino flux (16) generated by AQNs could be the same order of magnitude as the dominant ${ }^{8} B$ solar contributor $\Phi_{\nu_{e}} \simeq 5 \times 10^{6}\left(\mathrm{~cm}^{-2} \mathrm{~s}^{-1}\right)$ in this energy band. It is not presently ruled out by any experiment. The key point here is that the AQN-induced neutrino flux (16) is the subject of the annual modulation, as it has an inherent DM origin. Therefore, it could be the source of the observed DL modulation signal.

\section{Neutrino-induced neutrons}

The third element of proposal (1) is the liberation of the neutrons from surrounding rocks due to the coupling with neutrinos. The intensity of these liberated neutrons is the subject of annual modulation, as the corresponding neutron intensity is proportional to the neutrino flux (16), which itself is directly proportional to the DM flux in the form of the AQNs according to Eq. (9).

The idea that the surrounding neutrons might be the origin of the DL modulations has been suggested previously in a number of papers; see [7-10]. This proposal, of course, remains a subject of debate, as the DL Collaboration rejects the idea that surrounding neutrons could play any essential role in their observations [2]. We will make some comments within the AQN scenario in Sec. IV to address this question.

In the present subsection, we elaborate on the third element of our proposal (1) by estimating the neutron flux which is induced by neutrinos. The crucial difference with previous proposals [7-10] is that the neutrino-induced neutron flux (17) manifests itself with proper annual DM modulation [5,6] characterized by the expected phase $t_{0} \simeq 0.4 \mathrm{yr}$ corresponding approximately to June 1 for the standard halo model.

We use conventional expressions from Refs. $[2,10]$ for cross section $\sigma$ and the number density $n \simeq 10^{29} \mathrm{~m}^{-3}$ of the target to make a straightforward comparison with the previous estimates:

$R_{\nu}^{\mathrm{AQN}} \simeq \frac{d N_{\nu}}{d t d A} \sigma(\mathrm{nV}) \simeq 10^{-36} \cdot \kappa_{\nu} \cdot(\mathrm{nV})\left[\frac{\text { neutron }}{\mathrm{s}}\right]$, 
where we used the AQN-induced neutrino flux (16) and cross section $\sigma \simeq 10^{-41} \mathrm{~cm}^{2}$ for the neutrino-induced neutron spallation for the ${ }^{208} \mathrm{~Pb}$ target. The effective volume $V$ entering Eq. (17) will be discussed later in the text. The AQN-induced neutron rate production per unit volume can be represented as follows:

$$
r_{\nu}^{\mathrm{AQN}}=\frac{R_{\nu}^{\mathrm{AQN}}}{V} \simeq 10^{-2} \cdot \kappa_{\nu}\left[\frac{\text { neutron }}{\mathrm{day} \cdot \mathrm{m}^{3}}\right] .
$$

This rate is approximately one order of magnitude lower if $\kappa_{\nu} \simeq 1$ in comparison with corresponding estimates adopted in Refs. $[2,10]$ for the neutron's rate induced by the solar neutrinos. It could be the same order of magnitude as used in Refs. [2,10] if $\kappa_{\nu} \simeq 10$; see estimate (13). We return to the significance of estimate (18) later in the text. The only comment we would like to make here is that the typical kinetic energy of the neutrons liberated by this mechanism will be in the $10^{2} \mathrm{keV}$ range; see estimate (19) below.

Indeed, the first thing to notice is that the typical neutrino energy (13) is well above the neutron emission threshold $E_{\nu}>7.37 \mathrm{MeV}$ for the ${ }^{208} \mathrm{~Pb}$ target such that neutron spallation is kinematically allowed. Furthermore, if one assumes that momentum resulting from the neutron spallation is mostly transferred to the liberated neutron, such that $\boldsymbol{p}_{n} \simeq\left(\boldsymbol{p}_{\nu}^{\prime}-\boldsymbol{p}_{\nu}\right)$, the kinetic energy of the neutron can be estimated as follows:

$$
E_{n} \simeq \frac{\boldsymbol{p}_{n}^{2}}{2 m_{n}} \sim 10^{2} \mathrm{keV}
$$

One should also add that there is a sharp cutoff of the order of $\sim 10^{2} \mathrm{keV}$ for the neutron's energy (19) produced by this mechanism. It is determined by the neutrino energy (13) in the $15 \mathrm{MeV}$ range, which itself is kinematically bound from above by corresponding NG masses in the CS phase as reviewed in Appendix A. The presence of such a sharp cutoff will be an important element in our arguments supporting the proposal (1).

What do the neutrons, characterized by the rate (18) and energy (19), do if they enter the DL detector? This is the subject of the next subsection.

\section{DL modulation (1) due to the neutrons}

The fourth element of proposal (1) represents the most controversial portion of our analysis. We shall try to argue that neutrons characterized by the flux (18) and energy (19) may serve as the source of the observed DL annual modulation. The corresponding computations are very hard to carry out, as they are inevitably based on nuclear physics of a large number of very complicated systems. Fortunately, there are many specific experiments and tests which can be, in principle, performed, to be discussed in Secs. IV and V. These tests can support or rule out the proposal (1).
The starting point is the standard formula for energy transfer $\Delta E$ as a result of elastic $2 \rightarrow 2$ scattering when a target of mass $m_{2}$ is at rest, struck by a particle with mass $m_{1}$ with kinetic energy $E_{n}$ :

$$
\Delta E=2 E_{n} \frac{m_{1} m_{2}}{\left(m_{1}+m_{2}\right)^{2}}\left(1-\cos \theta_{\mathrm{CM}}\right) .
$$

The entire section of Ref. [7] was devoted for an explanation of numerous uses and misuses of this formula in different circumstances. We agree with most of the comments, careful explanation of misconceptions, and detailed analysis of the neutron-nuclear interaction given by Ralston in Ref. [7]. We defer our specific comments within the present context until Sec. IV.

Now we want to make a numerical estimate for the recoil energy using expression (20) and identifying $m_{1}$ with the neutron characterized by the kinetic energy (19), while $m_{2} \simeq 23 m_{1}$ with the lightest ${ }^{11} \mathrm{Na}$ nucleon from the DL detector consisting of 25 radio-pure NaI crystal scintillators:

$$
\Delta E=8.6 \mathrm{keV} \quad\left(1-\cos \theta_{\mathrm{CM}}\right) .
$$

The significance of this estimate is hard to overstate, as it unambiguously shows that the recoil energy cannot exceed the value (21), which is amazingly close to the $6 \mathrm{keV}$ cutoff observed by DL. Furthermore, the scale (19) was not invented for the specific proposal (1), in contrast with many different WIMP-based suggestions to fit the observed DL modulations. Rather, this scale is entirely determined by the cutoff in neutrino energy (13), which is itself unambiguously fixed by typical NG masses in the CS phase. All these scales have been known for quite some time, as reviewed in Appendix A.

The key element of our proposal (1) is that the flux of the induced neutrons (18) with kinetic energy (19), which eventually generates the signal in the DL detector with recoil energy (21), is the subject of annual modulation because all the intensities are proportional to DM velocity $\left\langle v_{\mathrm{AQN}}\right\rangle$ as Eq. (9) states. Therefore, to describe the corresponding modulations, one can use a conventional formula $[5,6]$ :

$$
\left\langle v_{\mathrm{AQN}}(t)\right\rangle \simeq V_{\odot}+b V_{\oplus} \cos \omega\left(t-t_{0}\right),
$$

where $V_{\odot}=220 \mathrm{~km} \mathrm{~s}^{-1}$ is the Sun's orbital speed around the Galactic center, $V_{\oplus}=29.8 \mathrm{~km} \mathrm{~s}^{-1}$ is Earth's orbital speed around the Sun, $\omega=2 \pi \mathrm{yr}^{-1}$ is the angular frequency of the annual modulation, and $|b| \leq 1$ is the geometrical factor associated with the direction of $v_{\mathrm{AQN}}$ relative to the orbital plane of Earth, $t_{0} \simeq 0.4 \mathrm{yr}$ corresponding to June 1 . Hence, it is natural to expect that the modulation must be of the order of $\mathcal{O}\left(V_{\oplus} / V_{\odot}\right) \sim 10 \%$, as incoming flux of the AQN particle explicitly proportional 
to $\left\langle v_{\mathrm{AQN}}(t)\right\rangle$ according to Eq. (9). The corresponding value $\langle\dot{N}(t)\rangle$ enters the expression for the AQN-induced neutrino flux (14), eventually generating the neutrons (18).

The very hard and challenging question remains to be answered if this neutron intensity (17) is sufficient for the explanation of the observed DL modulation. To answer this question, one has to understand the numerical value for the effective volume $V$ entering formula (17). As we already mentioned, this is very complicated problem of nuclear physics as emphasized and nicely explained in Ref. [7].

Therefore, instead of theoretical speculations about the value for the effective volume entering formula (17), we reverse the problem and estimate the volume $V$ which would match the DL modulation. We suggest several experiments how this proposal (1) and large rate (17) with effective volume $V$ can be tested in Secs. IV and V.

The DL modulation amplitude in terms of counts per day (cpd) [4] reads

DL modulation rate $=(0.0103 \pm 0.0008) \frac{\mathrm{cpd}}{\mathrm{kg} \mathrm{keV}}$

This rate must be multiplied to $4 \mathrm{keV}$ for the (2-6) $\mathrm{keV}$ energy range and $250 \mathrm{~kg}$ to get the total modulation rate

$$
\text { DL total modulation } \simeq 10\left[\frac{\text { counts }}{\text { day }}\right] .
$$

On the other hand, assuming that $10 \%$ of neutrons (18) are the subject of annual modulation with proper phase $t_{0}$ as explained in Eq. (22), we arrive at the following estimate in terms of the required effective volume $V \equiv L^{3}$ which saturates the DL modulation:

AQN-induced modulation $\simeq \kappa_{\nu}\left[\frac{\text { neutrons }}{\text { day }}\right]\left(\frac{L}{10 \mathrm{~m}}\right)^{3}$.

A few comments are in order. First of all, parameter $\kappa_{\nu}$ was introduced as a number of neutrinos being produced as a result of annihilation of a single baryon charge; see (13). It could be as small as $\kappa_{\nu} \sim 1$, but it could be as large as $\kappa_{\nu} \sim 10$, being consistent with presently available constraints as mentioned at the end of Sec. III B. The basic reason for this uncertainty is that our system is the strongly coupled gauge theory, to be contrasted with conventional weakly coupled gauge theories when all computations are under complete theoretical control. In our system, it is very hard to predict realistic spectra and intensities for neutrino and antineutrino fluxes in the $15 \mathrm{MeV}$ energy range due to the variety of possible phases, high sensitivity to the parameters, and large number of possible decay channels producing neutrinos and antineutrons, as we discussed above. This deficiency in our computational power should not be treated as a weakness of the proposal. Instead, it should be considered as a consequence of the complexity of the system.

The observed rate (24) matches the AQN-induced modulation (25) if $\kappa_{\nu} \simeq 10$ and $L \sim 10 \mathrm{~m}$. This required length $L \sim 10$ is definitely much greater than the neutron's mean free path $\lambda \simeq 2.6 \mathrm{~m}$, which was extracted from the studies on the muon-induced background [72] and adopted by Refs. $[2,10]$ in the context of the present work on DL modulations. In Sec. IV, we comment on the consistency (25) with DL observations, while in Sec. V we make a few comments on the relation to other experiments. Also in Sec. V, we suggest possible tests (such as the measuring of the spatial directions of moving neutrons along with time modulation) which could support or rule out the proposal (1).

\section{COMMENTS ON DL ARGUMENTS}

The DL Collaboration, of course, discussed the possibility that their signal is associated with neutron flux (induced by muons or neutrinos or both). In fact, the entire paper [2] was devoted to the analysis of a possible role of neutrinoinduced and muon-induced neutrons. These possibilities were discarded in Refs. [1-4] based on the following arguments:

(1) modulation phase arguments;

(2) energy range arguments; and

(3) intensity arguments.

We want to make a few comments on each of the items from this list. We start with the modulation arguments, while the energy and intensity arguments will follow.

(1) Quote from Ref. [2]: “... It is worth noting that neutrons, muons and solar neutrinos are not competing background when DM annual modulation signature is investigated since in no case they can mimic this signature..." In the proposed scenario (1), this argument obviously does not apply, because both the neutrino flux (16) and the neutron flux (17) with typical energy (19) are automatically the subject of the annual modulation (22) with proper phase $t_{0} \simeq 0.4 \mathrm{yr}$ corresponding to June 1 . This is because the source of the modulation in this framework has a truly genuine DM origin represented by AQNs.

(2) DL has carried out comprehensive studies on the dependence of the annual modulation as a function of the energy interval. The claim is that the modulation is not observed above the energy $\sim 6 \mathrm{keV}$. In particular, the modulation amplitude for the energy above $6 \mathrm{keV}$ for the whole datasets (DAMA/NaI, DAMA/LIBRA-phase-1, and DAMA/LIBRAphase-2) is shown to be consistent with zero; see Fig. 11 in Ref. [4]. This property is indeed very hard to understand in terms of the conventional physics advocated in Refs. [7-10].

At the same time, this unique feature of the system (characterized by a sharp cutoff at $\sim 6 \mathrm{keV}$ ) 
automatically emerges in our framework. Indeed, the neutrino flux (16) with typical neutrino energy $E_{\nu} \sim$ $15 \mathrm{MeV}$ is determined in our framework by the NG masses in the CS phase; see (A3). The corresponding neutrino-induced neutron flux (17) is characterized by the typical energy (19) formulated in terms of $E_{\nu}$. The sharp cutoff for the recoil energy (21) in this framework (which falls into the proper $\sim 6 \mathrm{keV}$ energy range) is determined by (19), which is essentially determined by the NG masses in the CS phase as reviewed in Appendix A. One should emphasize that all these energy scales have not been specifically invented in this work to explain the observed DL modulations with (1-6) keV energy; rather, the relevant energy scales have been established long ago in unrelated studies for different purposes in a different context.

(3) The neutrino flux (16) originated from AQNs in this framework is lower than the background solar neutrino flux $\Phi_{\nu_{e}} \simeq 5 \times 10^{6} \mathrm{~cm}^{-2} \mathrm{~s}^{-1}$ for this energy band (originating from solar ${ }^{8} \mathrm{~B}$ ) at least by a factor of 5 for $\kappa_{\nu} \simeq 10$, as mentioned at the end of Sec. III

B. The corresponding neutrino-induced neutron flux (17) also must be lower in comparison with the intensity of neutrons induced by the solar neutrinos. However, the key point is that this subdominant neutron component is originated from AQNs and, therefore, is the subject of conventional DM annual modulation (22) with proper phase $t_{0}$.

Is the corresponding neutrino-induced neutron's intensity sufficient ${ }^{9}$ to explain DL modulation? DL argued that the answer is "no" [1-4]. However, the DL arguments on the neutron's intensity were challenged in Ref. [7]. We have nothing new to add to these extensive discussions on the possible role of neutrino-induced neutrons. Instead of speculations about this very complex nuclear physics system with a complicated resonance structure, we suggest to test the proposal (1) by measuring the modulation, intensity, and directionality in coordinate space of the neutrons which are responsible for the recoil (21).

The subdominant flux of the AQN-induced neutrons can be, in principle, discriminated from the dominant components, including the solar neutrino-induced neutrons if the direction of the neutron's momentum and the modulation are measured. This is because the solar neutrinos are propagating from a single direction in the sky, while AQN-induced neutrinos (which have a truly genuine DM origin) are randomly distributed in space. This topic on possible tests of the proposal (1) represents the subject of Sec. V.

\footnotetext{
${ }^{9}$ As we already mentioned above, the question is essentially reduced to the quantitative understanding of the effective volume $V=L^{3}$ which enters (25). Our estimates from the previous section show that, if $L \simeq 10 \mathrm{~m}$, the AQN-induced rate (25) matches the observed rate (24) with $\kappa_{\nu} \simeq 10$.
}

\section{RELATION TO OTHER EXPERIMENTS AND POSSIBLE FUTURE TESTS}

This section is separated into three different subsections. First, in Sec. VA, we make a few comments on the previous experiments which exclude the DL signal if interpreted in terms of WIMP-nuclei interactions. We continue with a more recent analysis in Sec. VB, where we make a few comments on some recent experiments designed to reproduce (or rule out) the DL signals. Finally, in Secs. V C and $\mathrm{V} D$, we offer a few novel possible tests which can support or refute the proposal (1) explaining the DL signal in terms of the AQN-induced neutrons. In particular, in Sec. V D we propose to study a specific correlation between the amplification in the AQN-induced neutron flux and the impulses of the infrasound and weak seismic waves.

\section{A. Previous experiments}

We start with a few comments on the experiments which exclude the DL results. The corresponding collaborations include but are not limited to CDEX [73], CDMS- II [74,75], EDELWEISS-II [76], LUX [77], SuperCDMS [78], XENON10 [79], XENON100 [80], and CoGeNT [81]. The main claim of these collaborations can be formulated as follows: If DL modulation is interpreted in terms of WIMP-nuclei interactions with given $\sigma$ and given $m_{\text {WIMP }}$, then the DL signal is excluded with a very high level of confidence [82].

From the perspective of the proposal (1), there could be a number of reasons why DL observes the signal while other collaborations do not. First of all, most of the collaborations (with a few exceptions such as CoGeNT [81] and CDMS- II [75]) did not carry out dedicated studies on the time modulation, which was the crucial ingredient in DL arguments. From the AQN perspective, the time modulation is the key element when the subdominant neutron flux can manifest itself if proper time modulation studies are performed.

Another reason (for negative results) could be related to different neutron shields used by different collaborations. We refer to the paper by Ralston [7], where the subject on the complex behavior of neutrons in a complicated environment is nicely presented. This analysis obviously shows that even minor differences in neutron shields may have dramatic effects and drastically change the impact of the neutron's background.

Yet one more reason, probably the most important one in the context of the present work, is as follows. The AQNinduced neutrons with energy (19) are scattering off $\mathrm{Na}$ in the DL experiment, generating recoil energies which fall into the (2-6) $\mathrm{keV}$ bin according to Eq. (21). The recoil energies for heavier targets such as xenon or germanium in different experiments could be below the threshold, because the energies of the time-modulated neutrons (19) are bound from above with a cutoff being determined 
by energies of the AQN-induced neutrinos. The energies of these neutrinos are also bound from above and cannot exceed (13), as they are determined by NG masses in the CS phase. As we already mentioned previously, all these energy scales in proposal (1) have not been invented to fit the DL signals. Rather, the relevant energy scales have been established long ago in unrelated studies for different purposes in a different context.

\section{B. Recent activities}

Now we want to make a few comments on recent dedicated experiments which were specifically designed to test the DL annual modulation signal. It includes COSINE-100 [16-18], ANAIS-112 [19], and CYGNO [20] experiments. We also want to mention other types of experiments [83-85] which were not originally designed to test the DL annual modulation signal. However, their capabilities to measure the directionality could play a decisive role in detecting the DM signals. To be more specific, we choose to mention these experiments due to the following reasons.

The COSINE-100 experiment was mostly motivated by DL annual modulation. The aim of the collaboration is to reproduce (or refute) the signal and to search for a possible origin for the modulation, if observed. The COSINE-100 Collaboration uses the same target medium (sodium iodide) which is the crucial element in the context of the present proposal (1), as recoil energies fall into the (2-6) $\mathrm{keV}$ bin in our scenario according to Eq. (21). Presently, the COSINE100 data are consistent with both a null hypothesis and a DL (2-6) $\mathrm{keV}$ best fit value with $68 \%$ confidence level. More data are obviously needed. It is important that COSINE-100 is planning to measure the neutron's intensity and neutron's modulation [17], in which case COSINE-100 would know if the possible modulation is due to the neutrons. ${ }^{10}$ It is obviously the key element of the proposal (1) based on the subdominant AQN-induced component of neutrons which, however, manifests itself by annual modulation.

The ANAIS-112 Collaboration also uses the same target medium as DL and COSINE-100. ANAIS-112 has recently published the first results on annual modulation [19]. Their best fits are incompatible at $2.5 \sigma$ with the DL signal. The goal is to reach the sensitivity at $3 \sigma$ level in $5 \mathrm{yr}$. As the ANAIS-112 Collaboration uses the same target material, our comments from the previous paragraph in the context of the present proposal (1) also apply to the ANAIS-112 experiment, especially as ANAIS-112 and COSINE-100 agreed to combine their data.

The CYGNO proposal [20] is different from the COSINE-100 and the ANAIS-112 experiments due to

\footnotetext{
${ }^{10}$ I thank G. Adhikari for answering a large number of my questions during the PATRAS-2019 axion meeting about the future plans of the collaboration.
}

the capability to measure the directionality, which is the key element of the CYGNO [20] proposal. It is important that it will be located at the same site (LNGS) where DL is located. Therefore, the neutron flux must be the same, including the subdominant AQN-induced component (25) which is the subject of annual modulation. The collaboration is planning to measure (initially) the neutron flux and its modulation without neutron shielding. ${ }^{11}$ Such measurements may play a key role in supporting (or ruling out) the proposal (1), because the AQN-induced neutrons are responsible for the recoil (21). The collaboration is also planning in the future to reach the neutrino floor by measuring the neutrinos and their directions. In particular, CYGNO could discriminate the neutrinos from the Sun by identifying their directions. Furthermore, the CYGNO instrument will be capable to determine nuclear recoil directions, which would allow the collaboration to discriminate WIMP-like DM from AQN-induced events (1). Such measurements, if successful, would obviously play an important role in supporting (or ruling out) the proposal (1). In addition to that, the dominant solar neutrinos in the energy range $E_{\nu} \lesssim 12 \mathrm{MeV}$ could be discriminated from subdominant $\mathrm{AQN}$-induced neutrinos (A3). Furthermore, neutrinos in the energy band $E_{\nu} \geq 12 \mathrm{MeV}$ cannot be originated from the Sun at all as a result of the ${ }^{8} B$ threshold. The discovery of such neutrinos and measuring of their annual modulation with an intensity in the range (16) would be enormous support for the proposal (1), as the flux of the atmospheric neutrino is at least 3 orders of magnitude lower than (16).

We also want to mention several other experiments [83-85] which are capable to measure the directionality. The idea is to use carbon nanotube arrays or graphene layers to measure the directionality of the DM signals. As we mentioned above, measurements of the directionality could play a decisive role in detecting the DM signal.

\section{Possible future tests}

We already mentioned in Sec. V B a few possible tests which can support or rule out the proposal (1) with existing or planning experiments: COSINE-100 [16], ANAIS-112 [19], and CYGNO [20]. In this subsection, we want to mention a specific for the AQN framework phenomenon when the intensity of the AQN-induced neutrinos may be amplified by a very large factor (up to $10^{4}$ ) which greatly increases the chance for discovery of such AQN-induced neutrons which always accompany the neutrinos according to Sec. III C. Therefore, this neutrino amplification factor will be obviously accompanied by the corresponding amplification of the neutrino-induced neutron flux (17) and (18).

\footnotetext{
${ }^{11}$ I thank Elisabetta Baracchini for answering a large number of my questions during the PATRAS-2019 axion meeting about the future plans of the collaboration.
} 
The idea was originally formulated for the axions in Ref. [63], and the effect was coined as the "local flash." The computations can be easily generalized for the neutrinos in straightforward way; see Appendix B for some technical details. It can be explained as follows.

If the AQN hits the surface at distance $d \ll R_{\oplus}$ from the detector, the short-lasting flash occurs with amplification factor $A_{\nu}(d)$ measuring the relative short-lasting spark of the neutrino flux with respect to the neutrino flux (16) computed by averaging over the entire Earth's surface over a long period of time. The amplification $A_{\nu}(d)$ is highly sensitive to distance $d$ and can be approximated as follows [see (B4) for the derivation]:

$$
A_{\nu}(d) \simeq \frac{1}{\langle\dot{N}\rangle\langle\Delta t\rangle}\left(\frac{R_{\oplus}}{d}\right)^{2}
$$

One should note that the correction to the neutrino flux (B3) due to the traversing of a nearby AQN depends on unknown parameter $\kappa_{\nu}$. However, the relative amplification $A_{\nu}(d)$ with respect to the averaged neutrino flux (16) does not depend on $\kappa_{\nu}$ as Eq. (26) states. In formula (26), $\langle\dot{N}\rangle$ is determined by Eq. (9), while $\langle\Delta t\rangle \simeq 2 R_{\oplus} /\left\langle v_{\mathrm{AQN}}\right\rangle$ is the time for the AQN to cross Earth averaged over the entire ensemble of AQN trajectories traversing Earth.

As one can see from Eq. (26), a huge amplification may indeed occur for $d \ll R_{\oplus}$. However, the probability for such an event to happen is very tiny and can be estimated as

$$
\text { event rate } \simeq \frac{1}{\left[\langle\dot{N}\rangle\langle\Delta t\rangle^{3}\right]^{1 / 2}} \cdot \frac{1}{A_{\nu}^{3 / 2}}
$$

see Appendix B for details. The "local flash" lasts for a short period of time which can be estimated as follows:

$$
\tau \simeq \frac{2 d}{\left\langle v_{\mathrm{AQN}}\right\rangle} \simeq\left(\frac{\langle\Delta t\rangle}{\langle\dot{N}\rangle}\right)^{1 / 2} \frac{1}{A_{\nu}^{1 / 2}}
$$

We summarize in Table I a few choices of time duration $\tau$ and the event rate as a function of amplification factor $A_{\nu}$. In particular, it would be a daily short-lasting "flash" when the intensity of the subdominant AQN-induced neutrino component (16) is amplified by factor $A_{\nu}(d) \simeq 10^{2}$ such

TABLE I. Estimations of local flashes for different $A_{\nu}$, adopted from Ref. [63]: the time duration and the corresponding event rate.

\begin{tabular}{lcc}
\hline \hline$A_{\nu}$ & $\tau$ (time span) & Event rate \\
\hline 1 & $10 \mathrm{~s}$ & $0.3 \mathrm{~min}^{-1}$ \\
10 & $3 \mathrm{~s}$ & $0.5 \mathrm{hr}^{-1}$ \\
$10^{2}$ & $1 \mathrm{~s}$ & $0.4 \mathrm{day}^{-1}$ \\
$10^{3}$ & $0.3 \mathrm{~s}$ & $5 \mathrm{yr}^{-1}$ \\
$10^{4}$ & $0.1 \mathrm{~s}$ & $0.2 \mathrm{yr}^{-1}$ \\
\hline \hline
\end{tabular}

that it becomes the dominant one for a short period of time lasting for about $1 \mathrm{~s}$.

An important lesson to be learned from these estimates is as follows. A subdominant neutrino flux induced by AQNs may become a dominant portion of the neutrinos, overpassing the solar neutrino flux in this energy band for a very short period of time. Needless to say, this AQN-induced neutrino flux (16) and the corresponding neutron flux (17) are also the subject of annual (22) and daily modulations, ${ }^{12}$ similar to the ones studied for the axion search experiments [63]. The measure of directionality and modulation as described in Sec. VB may help to discriminate this subdominant AQN-induced neutrino flux from the dominant solar ${ }^{8} B$ component.

\section{Search for correlations with other AQN-induced phenomena}

The flux (8) suggests that the AQNs hit Earth's surface with a frequency approximately once a day per $100^{2} \mathrm{~km}^{2}$ area, which is precisely the source for a short-lasting amplification in the neutrino production discussed in Sec. V C. It is important to emphasize that such events occur along with other processes which always accompany the AQN propagation through the atmosphere and Earth's underground. Therefore, there will be always some correlations between the amplifications in the neutrino flux and other associated phenomena in the vicinity of the area where an AQN event occurs.

For example, if the DM detector (sensitive to neutrinoinduced neutrons such as DL) and an axion search detector are localized close to each other on a distance $d_{\nu a} \sim d$, there will be the axion signal due to the amplification $A_{a}$ and the neutrino-induced neutron signal due to the amplification $A_{\nu}$. These signals must be correlated in the form of two almost simultaneous short-lasting sparks between these two signals in two different detectors. The observation of these cross-correlated signals (collected during a long period of time and by measuring the directionality in the DM detector to discriminate the background) would unambiguously support the proposal (1) on the nature of the observed DL modulation signal. A similar cross-correlation between different synchronized axion stations from a global network as suggested in Ref. [86] and a nearby neutrino detector would also strongly support the proposal (1).

In particular, the position of the Center for Axion and Precision Physics Research located at Daejeon and COSINE-100 detector located at the Yangyang Underground Laboratory in South Korea obviously satisfy the criteria $d_{\nu a} \ll 0.1 R_{\oplus}$ when strongly correlated amplifications for $A_{\nu}$ and $A_{a}$ may occur in both detectors almost

\footnotetext{
${ }^{12}$ Daily modulations with an intensity around (1-10)\% are similar in magnitude as annual modulations. These daily modulations are unique for this type of DM and not shared by any other DM models; see [63] for the details.
} 
simultaneously with a time delay of the order of a few seconds.

Another correlation which is worthwhile to study can be explained as follows. It has been recently argued that the AQN propagating in Earth's atmosphere and underground emits infrasound and weak seismic waves [87]. In fact, one such event, according to Ref. [87], occurred on 31 July, 2008. It was properly recorded by the dedicated Elginfield Infrasound Array (ELFO) near London, Ontario, Canada, and corresponds to a relatively large nugget ${ }^{13}$ with $B \simeq 10^{27}$ if interpreted as an AQN event [87]. The infrasound detection was accompanied by nonobservation of any meteors by an all-sky camera network. The impulses were also observed seismically as ground coupled acoustic waves around southwestern Ontario and northern Michigan. The estimates [87] for the infrasonic frequency $\nu \simeq 5 \mathrm{~Hz}$ and overpressure $\delta p \sim 0.3 \mathrm{~Pa}$ are consistent with the ELFO record. A detection strategy has been also proposed in Ref. [87] for a systematic study to search for such events originating from much smaller and much more common AQNs with typical $B \simeq 10^{25}$ by using distributed acoustic sensing (DAS).

Our original remark here is that the amplification in the neutrino flux (and corresponding enhancement in the neutrino-induced neutrons) as discussed in Sec. V C must be accompanied by infrasonic and weak seismic waves which can be studied by the DAS instruments implemented in networks of optical-fiber telecommunication cables. The observation for such correlations, if successful, is absolutely unique to the AQN framework and would obviously play an important role in supporting of the proposal.

In particular, DL (and future CYGNO detector) is located at the LNGS site with a large number of seismic detectors located in the same area. The proposal is to search for the correlations between the enhanced flux of neutrons and weak seismic and infrasound events with the delay measured in a fraction of a second depending on a precise localization of the seismic detectors. The measurements of the directionality by CYGNO would be the key element in establishing such a correlation.

\section{CONCLUDING COMMENTS}

The main results of our work can be summarized as follows.

We argued that the annual modulation observed by DL might be explained as a result of the AQN-induced neutrons through the chain (1). In this framework, the annual modulation has a truly genuine DM origin, though it is manifested indirectly. We also argued that the recoil energy

\footnotetext{
${ }^{13}$ The frequency of appearance for such large nuggets is very tiny according to Eq. (6). This explains why such events occur approximately once every $10 \mathrm{yr}$ instead of observations of them once a day, which would be the case for much more common nuggets with $B \sim 10^{25}$.
}

must have a sharp cutoff at $\sim 6 \mathrm{keV}$ consistent with the observed DL signal. We proposed specific tests which can support or rule our the proposal (1); see Sec. V C. We proposed to study a specific correlation between the amplification in the AQN-induced neutron flux and the impulses of the infrasound and weak seismic waves, which, if found, would strongly support our proposal; see Sec. V D.

Why should we consider this AQN model seriously? There are a number of reasons. Originally, this model was invented to explain the observed relation $\Omega_{\mathrm{DM}} \sim \Omega_{\mathrm{visible}}$ and the baryon asymmetry of the Universe as two sides of the same coin, when the baryogenesis framework is replaced by a "charge separation" framework, as reviewed in Sec. II. After many years since its original formulation, this model remains consistent with all available cosmological, astrophysical, satellite, and ground-based constraints, where AQNs could leave a detectable electromagnetic signature. Furthermore, it is shown that AQNs can be formed and can survive the unfriendly environment during the evolution of the early Universe, such that they are entitled to serve as DM candidates. Finally, the same AQN framework may also explain a number of other (naively unrelated) observed phenomena such as the excess of galactic diffuse emission in different frequency bands, the so-called primordial lithium puzzle and the Solar corona mystery, and the seasonal variations observed by XMM-Newton observatory, to name just a few; see Sec. I for the references.

We want to emphasize that all these cosmological puzzles mentioned in Sec. I could be resolved within the AQN framework with the same set of physical parameters being used in the present work on the explanation of the DL modulation signal.

The observation of the subdominant AQN-induced neutrons by measuring the directionality and modulation as discussed in Sec. V B would be a direct manifestation of the AQN dark matter model. The observation of a variety of amplifications as discussed in Sec. V C would be also a strong support for the proposal (1). Finally, the recording of the correlations between the AQN-induced neutrino amplification and the impulses of the infrasound and weak seismic waves would strongly support our proposal as argued in Sec. V D. We finish this work on this positive and optimistic note.

\section{ACKNOWLEDGMENTS}

I am thankful to G. Adhikari for answering my questions on the present status and future plans for the COSINE-100 Collaboration (see footnote 10) and Elisabetta Baracchini for elaboration on future plans for the CYGNO Collaboration (see footnote 11) during the PATRAS-2019 axion meeting in Freiburg. I am thankful to Maria Martinez [ANAIS-112] and Elisabetta Baracchini [CYGNO] for correspondence. I am also thankful to Hyunsu Lee [COSINE] and Maria Luisa Sarsa [ANAIS-112] for discussions 
during the "Conference on Dark World" in Daejeon, Korea, where this work was presented. This research was supported in part by the Natural Sciences and Engineering Research Council of Canada.

\section{APPENDIX A: NEUTRINO SPECTRUM FROM THE AXION QUARK NUGGETS}

The main goal of this Appendix is to give a short overview of the basic results from Ref. [65] regarding the neutrinos emitted by AQNs captured by the Sun. The paper [65] was written in response to the claim made in Ref. [64] that dark matter in the form of AQNs cannot account for more than $20 \%$ of the dark matter density. This claim was based on constraints on the neutrino flux in the (20-50) MeV range, where the sensitivity of underground neutrino detectors such as Super-Kamiokande have their highest signal-to-noise ratio.

However, the estimates [64] were based on an assumption that the annihilation processes between antimatter from AQNs and normal material from the Sun have the same spectral features as conventional baryon-antibaryon annihilations, which typically produce a large number of pions which eventually decay though an intermediate muon and, thus, generate a significant number of neutrinos and antineutrinos in the (20-50) MeV range.

However, as it has been argued in Ref. [65], the critical difference in the case of annihilation processes involving an antiquark nugget is that the annihilation proceeds within the CS phase, where the energetics are drastically different. The main point is that in most CS phases the lightest pseudo-Goldstone mesons (the pions and kaons) have masses in the $20 \mathrm{MeV}$ range [66,67], in huge contrast with the hadronic confined phase where $m_{\pi} \sim 140 \mathrm{MeV}$. As a result of this crucial difference, the decay of light pseudo-Goldstone mesons of the CS phase cannot produce neutrinos in the $20-50 \mathrm{MeV}$ energy range and are not subject to the SuperK constraints employed in Ref. [64]. Instead, the pseudo-Goldstone mesons of the CS phase produce neutrinos in the $15 \mathrm{MeV}$ range.

These unique spectral features of the neutrinos emitted by AQNs play the key role in our proposal, suggesting that the observed cutoff in DL modulations at $6 \mathrm{keV}$ is ultimately related to the cutoff in the neutrino energies at $15 \mathrm{MeV}$ emitted by AQNs. The emergence of this new $15 \mathrm{MeV}$ energy scale is the subject of the next subsections.

\section{Nambu-Goldstone modes in the CS phase}

There are many possible CS phases due to the generation of a gap $\Delta$ through different channels with slightly different properties. While the relevant physics is a part of the standard model, QCD with no free parameters, the corresponding phase diagram is still a matter of debate, as it strongly depends on the precise numerical value of the gap $\Delta$; see review articles $[66,67]$. For our purposes, though, the key characteristics are very much the same for all phases. Therefore, we limit ourself below to reviewing the most developed, the so-called color flavor locking (CFL) phase. The spontaneous breaking of chiral symmetry in color superconductors gives rise to low-energy pseudo-NambuGoldstone (NG) modes with similar quantum numbers to the mesons (pions, kaons, etc.). These objects, however, are collective excitations of the CS state rather than vacuum excitations as is the case for the conventional confined hadronic phase. The finite quark masses explicitly break chiral symmetry, giving rise to these "pseudo-NambuGoldstone" modes on the order of $20 \mathrm{MeV}$, in huge contrast with the hadronic confined phase where the lightest mass meson has $m_{\pi} \simeq 140 \mathrm{MeV}$.

To be more precise, we consider a large $\mu$ limit for which the masses and other relevant parameters in the CFL phase can be explicitly computed $[66,67]$ :

$$
\begin{array}{ll}
m_{\pi^{ \pm}}^{2} \simeq \frac{2 \bar{c}}{f_{\pi}^{2}} m_{s}\left(m_{u}+m_{d}\right), & f_{\pi}^{2} \sim \mu^{2}, \\
m_{K^{ \pm}}^{2} \simeq \frac{2 \bar{c}}{f_{\pi}^{2}} m_{d}\left(m_{u}+m_{s}\right), & \bar{c} \simeq \frac{3 \Delta^{2}}{2 \pi^{2}}, \\
m_{K^{0}}^{2} \simeq \frac{2 \bar{c}}{f_{\pi}^{2}} m_{u}\left(m_{d}+m_{s}\right) . &
\end{array}
$$

As one can see from Eq. (A1), the NG bosons are much lighter than in a vacuum. This is because their masses are proportional to $m_{q}^{2}$ rather than to $m_{q}$, as at zero chemical potential. As a result, the lightest NG meson, the kaon, has a mass in the range of 10-20 MeV depending on the precise value of $\Delta$ and $\mu \mathrm{MeV}$; see, for example, [67].

Another important difference between the NG modes in dense matter and in a vacuum is in the dispersion relations for the NG modes, which assume the following form (see, for example, [66]):

$$
\begin{aligned}
& E_{K^{ \pm}}=\mp \frac{m_{s}^{2}}{2 \mu}+\sqrt{v_{\mathrm{NG}}^{2} p^{2}+m_{K^{ \pm}}^{2}}, \\
& E_{K^{0}}=-\frac{m_{s}^{2}}{2 \mu}+\sqrt{v_{\mathrm{NG}}^{2} p^{2}+m_{K^{0}}^{2}}, \\
& E_{\bar{K}^{0}}=+\frac{m_{s}^{2}}{2 \mu}+\sqrt{v_{\mathrm{NG}}^{2} p^{2}+m_{K^{0}}^{2}}, \\
& E_{\pi^{ \pm}}=\sqrt{v_{\mathrm{NG}}^{2} p^{2}+m_{\pi^{ \pm}}^{2}}, \quad v_{\mathrm{NG}}^{2}=\frac{c^{2}}{3},
\end{aligned}
$$

such that the rest energy of the lightest NG mesons does not exceed the $10-20 \mathrm{MeV}$ range. In fact, $E_{K^{0}}$ may even vanish, in which case the $K^{0}$ field forms a condensate (the so-called CFL $K^{0}$ phase). In these formulas, $v_{\mathrm{NG}}$ deviates from the speed of light $c$ due to the explicit violation of the Lorentz invariance in the system such that the dispersion relations for all quasiparticles are drastically different from their counterparts in a vacuum. 
One should comment here that the dispersion relations for the NG modes within the antinuggets (which is most relevant for our purposes) can be obtained from Eq. (A2) by replacing $\mu \rightarrow-\mu$ such that the lightest NG states become the $\bar{K}^{0}$ and $K^{-}$for nuggets made of antimatter. This comment is important for identification of the neutrino and antineutrino spectra to be discussed in Appendix A 2.

\section{Neutrino emission from NG bosons}

The neutrino emission from CFL phase quark matter has been studied previously in a number of papers mostly in the context of the physics of neutron stars; see the original papers [88-90] and the review article [66].

In this subsection, we will overview the basic results of Ref. [65] on neutrino and antineutrino fluxes from the Sun. The main goal of Ref. [65] was to argue that the SuperKamiokande stringent constraint on antineutrino flux $\Phi_{\bar{\nu}_{e}}<$ (1.4-1.9) $\mathrm{cm}^{-2} \mathrm{~s}^{-1}$ at large energies $E>19.3 \mathrm{MeV}$ [69] (which played a key role in the analysis of Ref. [64]) does not apply to our case on neutrino and antineutrino production by AQNs, because the typical energies of neutrinos and antineutrinos will be much lower.

Indeed, the muons cannot be produced at all in the CFL phase for purely kinematical reasons. Therefore, the energetic antineutrinos which are normally produced in the $\mu^{ \pm} \rightarrow e^{ \pm} \nu_{e} \nu_{\mu}$ decay channels are not produced in the CS matter. This is the crucial point of the arguments presented in Ref. [65].

In the simplest possible scenario (which was adopted in Ref. [65]), the majority of neutrinos will be emitted by the lightest NG bosons, in which case the energy of the emitted neutrinos does not normally exceed $15 \mathrm{MeV}$ for the CFL phase, because the lightest NG bosons do not normally exceed a mass in the $30 \mathrm{MeV}$ range as mentioned in Appendix A 1. This is a very basic and very generic feature of the CS phase. We postpone the important discussions on basic features of the neutrinos emitted by the quarks in CS phases to Appendix A 3. Below, we list a few features on the neutrino spectrum if it is saturated exclusively by NG bosons:

(1) A specific choice of $\mu$ and $\Delta$ determines the basic mass scales for the light NG modes, which eventually determine the $\nu$ spectrum.

(2) It is normally assumed that a required value for $\mu$ for CFL to be realized is $\mu \simeq 400 \mathrm{MeV}$. The corresponding value for $\Delta$ is estimated in this case as $\Delta \simeq 100 \mathrm{MeV}$ in the CFL phase; see Ref. [66]. Such a large value of $\mu$ can be indeed reached in the AQN's core as recent numerical studies suggest; see Fig. 2 in Ref. [46].

(3) The neutrino spectrum is qualitatively different from that of the antineutrinos, because the annihilation occurs not in a vacuum but in a dense CS state with $\mu \simeq 400 \mathrm{MeV}$.
(4) Larger chemical potentials $\mu$ generally lead to even lighter NG modes, while the masses increase with the size of the gap $\Delta$ as Eqs. (A1) and (A2) state.

We conclude this subsection with the following generic comment. The main goal of Ref. [65] was to argue (in a simplified setting) that the neutrino's energies in the AQN framework are typically in the $15 \mathrm{MeV}$ range (in contrast with the 20-50 MeV range as assumed in Ref. [64]) such that the stringent constraint from SuperK [69] does not apply.

\section{Fermion excitations and neutrino's production in CS phases}

As we already mentioned, the neutrino emission from CS phase quark matter has been studied previously in a number of papers mostly in the context of the physics of neutron stars; see the review article [66]. We cannot literally apply the machinery developed in previous studies, because all excitations (such as NG bosons) in our case are produced not in a thermally equilibrium system when the density of the excitations is unambiguously determined by the temperature. Instead, all excitations in our scenario are produced as a result of rare annihilation events. These annihilation events excite the NG bosons as well as fermion excitations, which may decay by emitting neutrinos. The coefficients $\kappa_{\nu}$ and $\kappa_{\bar{\nu}}$ entering Eq. (16) precisely correspond to this mechanism of the neutrino production.

In this subsection, we want to overview some features of the fermion excitations of the CS phases which may be the dominant contributors to the neutrino fluxes. This is because the NG bosons which are produced as a result of annihilation events in the CS phase cannot leave the system and consequently decay to emit neutrino, as they must stay inside of the nuggets. It should be contrasted with the hadronic phase when pions and kaons (produced as a result of, e.g., $p \bar{p}$ annihilation) decay to muons and neutrinos in a vacuum. Therefore, the NG bosons in CS phases are likely to be absorbed by fermion excitations (if kinematically allowed), which consequently decay to neutrinos.

We start our overview by mentioning some CS phases which support low-energy fermion excitations. Detailed discussions can be found in Ref. [66]. First of all, the socalled 2SC phase (when two out of three colors and flavors are paired and condensed) supports unpaired modes which could be light and couple to NG bosons. Another phase which also supports the light fermion excitations is the socalled CFL- $K^{0}$ phase when $K^{0}$ energy vanishes according to Eq. (A2). For antinuggets, this phase corresponds to $\bar{K}^{0}$ condensation. In both cases (CFL and CFL- $K^{0}$ ), the gap of the excitations decreases with increases of $p_{F}$ such that the gaps for $p$ and $n$ become lower. Indeed, $\Delta_{p, n}=\Delta-\frac{m_{s}^{2}}{2 p_{F}}$ such that $p, n$ fermion excitations could become completely ungapped. If this happens, these modes may become the dominant producers of the neutrinos. 
Indeed, in unpaired quark matter, neutrino emissivity is dominated by the direct Urca processes such as $d \rightarrow u+e^{-}+\bar{\nu}_{e}$. In the case of antinuggets, it should be replaced by antiquarks with emission of neutrino $\nu_{e}$ and positron $e^{+}$with the energy determined by the energy of the fermion excitation, which itself assumes the energy of the order of the lightest NG mode according to analysis of Appendix A 2.

If this process indeed becomes the dominant mechanism of the neutrino emission from AQNs, then one should expect that

$$
\kappa_{\nu} \gg \kappa_{\bar{\nu}}, \quad E_{\nu} \lesssim 15 \mathrm{MeV}, \quad \kappa_{\nu} \gtrsim 1,
$$

which is assumed to be the case as Eq. (13) states.

\section{APPENDIX B: LOCAL FLASHES}

In this Appendix, we generalize our axion studies [63] to include the neutrinos into consideration, similar to what we have done in Sec. III B. The main topic for the present studies is the enhancement effect and great amplification of the axion density which was coined as a "local flash" in Ref. [63]. It occurs on rare occasions when an AQN hits (or exits) Earth's surface in the vicinity of an axion search detector. In this Appendix, we generalize the arguments of Sec. III B to estimate a similar local flash for neutrinos.

We follow the same logic of Ref. [63] and consider a case when an AQN is moving in a distance $d$ close enough to the detector, as shown in Fig. 1. The total number of emitted neutrinos per unit area within $z \in\left[-z_{\text {cut }}, z_{\text {cut }}\right]$ as a result of passage of the AQN at distance $d$ from an observer is given by

$$
\begin{aligned}
\Delta \frac{\mathrm{d} N_{\nu}}{\mathrm{d} A} & =\frac{1}{4 \pi} \int_{-z_{\mathrm{cut}}}^{z_{\mathrm{cut}}} \frac{\kappa_{\nu}}{z^{2}+d^{2}} \frac{\mathrm{d} m_{\mathrm{AQN}}(z)}{m_{p}} \\
& \simeq \frac{\beta}{2 \pi d} \frac{\kappa_{\nu}}{v_{\mathrm{AQN}}} \frac{\dot{m}_{\mathrm{AQN}}}{m_{p}},
\end{aligned}
$$

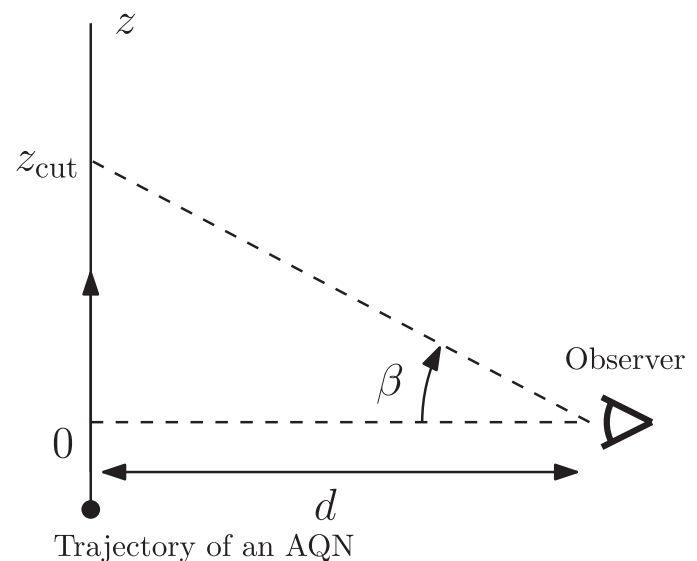

FIG. 1. A local flash occurs for a short period of time $\tau$ when an AQN moves at distance $d \ll R_{\oplus}$, adopted from Ref. [63]. where $\beta$ is the angle related to $z_{\text {cut }}$ as shown in Fig. 1, and we used formula (13) which defines the coefficient $\kappa_{\nu}$ as the number of neutrinos produced due to the annihilation of a single baryon charge. In obtaining Eq. (B1), the integration $\mathrm{d} z$ is replaced by integration $\mathrm{d} m_{\mathrm{AQN}}$ :

$$
\mathrm{d} N_{\nu} \simeq \frac{\kappa_{\nu}}{m_{p}} \mathrm{~d} m_{\mathrm{AQN}}=\frac{\kappa_{\nu}}{m_{p}} \frac{\dot{m}_{\mathrm{AQN}}}{v_{\mathrm{AQN}}} \mathrm{d} z
$$

Now we can estimate the flux of neutrinos due to the passage of this nearby AQN as follows:

$$
\Delta\left(\frac{\mathrm{d} N_{\nu}}{\mathrm{d} A \mathrm{~d} t}\right) \simeq \frac{1}{\tau} \Delta\left(\frac{\mathrm{d} N_{\nu}}{\mathrm{d} A}\right)=\frac{\kappa_{\nu}}{m_{p}} \frac{\beta}{4 \pi d^{2}} \dot{m}_{\mathrm{AQN}},
$$

where we used expression (B1) and approximated $\tau \simeq$ $2 d / v_{\mathrm{AQN}}$ as a typical travel time for an AQN inside the interval $\left[-z_{\text {cut }}, z_{\text {cut }}\right]$.

We want to compare this local flash (B3) with the average flux (14) by introducing the amplification factor $A_{\nu}(d)$ defined as the ratio:

$$
A_{\nu}(d) \equiv \frac{\Delta\left(\frac{\mathrm{d} N_{\nu}}{\mathrm{d} A \mathrm{~d} t}\right)}{\left(\frac{\mathrm{d} N_{\nu}}{\mathrm{d} A \mathrm{~d} t}\right)} \simeq \frac{\beta}{\langle\dot{N}\rangle\langle\Delta t\rangle}\left(\frac{R_{\oplus}}{d}\right)^{2},
$$

where we approximated $\dot{m}_{\mathrm{AQN}} \simeq\left\langle\Delta m_{\mathrm{AQN}}\right\rangle /\langle\Delta t\rangle$. The physical meaning of $\langle\Delta t\rangle$ is the time duration of the AQNs being averaged over all trajectories and over the velocity distribution. The result (B4) does not depend on the neutrino's spectrum nor intensity. It does not include even parameter $\kappa_{\nu}$ and, in fact, identically coincides with the expression obtained for the axion local flash derived previously in Ref. [63]. It is an anticipated result, as all these numerical factors cancel out in the ratio (B4) as relative amplification factor (B4) is entirely determined by the dynamics of the AQNs, not the particles they emit as long as these particles are relativistic, which is the case for both species: the axions and neutrinos.

As the final expression (B4) for the neutrino amplification factor coincides with the corresponding expression for the axion [63], the consequences in both cases are the same, and we simply list them.

(1) For the typical ratio $\langle\dot{N}\rangle\langle\Delta t\rangle \sim 30$, where $\langle\dot{N}\rangle$ is given by Eq. (9) and $\beta \sim 1$, one can infer that an amplification becomes significant if $d \ll 0.1 R_{\oplus}$.

(2) The time duration $\tau$ of a local flash as a function of amplification $A_{\nu}$ :

$$
\tau \simeq \frac{2 d}{v_{\mathrm{AQN}}} \simeq\left(\frac{\langle\Delta t\rangle}{\langle\dot{N}\rangle}\right)^{1 / 2} \frac{1}{A_{\nu}^{1 / 2}},
$$

where, in the last step, we approximate $v_{\mathrm{AQN}} \simeq$ $2 R_{\oplus} /\langle\Delta t\rangle$ and assume $\beta \sim 1$ for simplicity. We summarize a few choices of time duration $\tau$ as a function of amplification factor $A_{\nu}$ in Table I. 
(3) The probability to observe an AQN for $z \leq d$ behaves as a simple area law:

$$
\operatorname{Prob}(z \leq d) \simeq\left(\frac{d}{R_{\oplus}}\right)^{2} \simeq \frac{1}{\langle\dot{N}\rangle\langle\Delta t\rangle} \cdot \frac{1}{A_{\nu}}
$$

where we use Eq. (B4) to express $d$ in terms of $A_{\nu}$.

(4) The event rate can be expressed in terms of amplification parameter $A_{\nu}$ :

$$
\text { event rate }=\frac{\langle\dot{N}\rangle \cdot \operatorname{Prob}(z \leq d) \cdot \tau}{\langle\Delta t\rangle} \simeq \frac{A_{\nu}^{-3 / 2}}{\sqrt{\langle\dot{N}\rangle\langle\Delta t\rangle^{3}}},
$$

where averages $\langle\dot{N}\rangle$ and $\langle\Delta t\rangle$ have been numerically computed for different size distribution models in Ref. [63].
[1] R. Bernabei et al., Eur. Phys. J. C 73, 2648 (2013).

[2] R. Bernabei et al., Eur. Phys. J. C 74, 3196 (2014).

[3] R. Bernabei et al., Bled Workshops Phys. 17, 1 (2016) [EPJ Web Conf. 136, 05001 (2017).

[4] R. Bernabei et al., Universe 4, 116 (2018) [Nucl. Phys. At. Energy 19, 307 (2018).

[5] K. Freese, J. A. Frieman, and A. Gould, Phys. Rev. D 37, 3388 (1988).

[6] K. Freese, M. Lisanti, and C. Savage, Rev. Mod. Phys. 85, 1561 (2013).

[7] J. P. Ralston, arXiv:1006.5255.

[8] D. Nygren, arXiv:1102.0815.

[9] K. Blum, arXiv:1110.0857.

[10] J. H. Davis, Phys. Rev. Lett. 113, 081302 (2014).

[11] V. V. Flambaum and A. R. Zhitnitsky, Phys. Rev. D 99, 023517 (2019).

[12] A. Zhitnitsky, J. Cosmol. Astropart. Phys. 10 (2017) 050.

[13] N. Raza, L. van Waerbeke, and A. Zhitnitsky, Phys. Rev. D 98, 103527 (2018).

[14] K. Lawson and A. R. Zhitnitsky, Phys. Dark Universe 24, 100295 (2019).

[15] S. Ge, H. Rachmat, M. S. R. Siddiqui, L. Van Waerbeke, and A. Zhitnitsky, arXiv:2004.00632.

[16] G. Adhikari et al. (COSINE-100 Collaboration), Nature (London) 564, 83 (2018); 566, E2 (2019).

[17] G. Adhikari et al. (COSINE-100 Collaboration), J. Instrum. 13, T06005 (2018).

[18] G. Adhikari et al. (COSINE-100 Collaboration), Phys. Rev. Lett. 123, 031302 (2019).

[19] J. Amar et al. (ANAIS-112 Collaboration), Phys. Rev. Lett. 123, 031301 (2019).

[20] CYGNUS Collaboration, arXiv:1901.04190.

[21] E. Witten, Phys. Rev. D 30, 272 (1984).

[22] E. Farhi and R. L. Jaffe, Phys. Rev. D 30, 2379 (1984).

[23] A. De Rujula and S. L. Glashow, Nature (London) 312, 734 (1984).

[24] J. Madsen, Lect. Notes Phys. 516, 162 (1999).

[25] A. R. Zhitnitsky, J. Cosmol. Astropart. Phys. 10 (2003) 010.

[26] R. D. Peccei and H. R. Quinn, Phys. Rev. D 16, 1791 (1977).

[27] S. Weinberg, Phys. Rev. Lett. 40, 223 (1978).

[28] F. Wilczek, Phys. Rev. Lett. 40, 279 (1978).

[29] J. E. Kim, Phys. Rev. Lett. 43, 103 (1979).
[30] M. A. Shifman, A. I. Vainshtein, and V. I. Zakharov, Nucl. Phys. B166, 493 (1980).

[31] M. Dine, W. Fischler, and M. Srednicki, Phys. Lett. 104B, 199 (1981).

[32] A. R. Zhitnitsky, Yad. Fiz. 31, 497 (1980) [Sov. J. Nucl. Phys. 31, 260 (1980)].

[33] K. Van Bibber and L. J. Rosenberg, Phys. Today 59, No. 8, 30 (2006).

[34] S. J. Asztalos, L. J. Rosenberg, K. van Bibber, P. Sikivie, and K. Zioutas, Annu. Rev. Nucl. Part. Sci. 56, 293 (2006).

[35] P. Sikivie, in Axions, edited by M. Kuster, G. Raffelt, and B. Beltrán, Lecture Notes in Physics Vol. 741 (Springer Verlag, Berlin, 2008), p. 19.

[36] G. G. Raffelt, in Axions, edited by M. Kuster, G. Raffelt, and B. Beltrán, Lecture Notes in Physics Vol. 741 (Springer Verlag, Berlin, 2008), p. 51.

[37] P. Sikivie, Int. J. Mod. Phys. A 25, 554 (2010).

[38] L. J. Rosenberg, Proc. Natl. Acad. Sci. U.S. A. 112, 12278 (2015).

[39] D. J. E. Marsh, Phys. Rep. 643, 1 (2016).

[40] P. W. Graham, I. G. Irastorza, S. K. Lamoreaux, A. Lindner, and K. A. van Bibber, Annu. Rev. Nucl. Part. Sci. 65, 485 (2015).

[41] A. Ringwald, in Proceedings of the Neutrino Oscillation Workshop (NOW2016), Otranto (Lecce, Italy) (SISSA, Trieste, Italy 2016), p. 81.

[42] I. G. Irastorza and J. Redondo, Prog. Part. Nucl. Phys. 102, 89 (2018).

[43] X. Liang and A. Zhitnitsky, Phys. Rev. D 94, 083502 (2016).

[44] S. Ge, X. Liang, and A. Zhitnitsky, Phys. Rev. D 96, 063514 (2017).

[45] S. Ge, X. Liang, and A. Zhitnitsky, Phys. Rev. D 97, 043008 (2018).

[46] S. Ge, K. Lawson, and A. Zhitnitsky, Phys. Rev. D 99, 116017 (2019).

[47] A. Zhitnitsky, Phys. Rev. D 74, 043515 (2006).

[48] K. Lawson, Phys. Rev. D 83, 103520 (2011).

[49] M. G. Aartsen et al. (IceCube Collaboration), Eur. Phys. J. C 74, 2938 (2014).

[50] K. Lawson, X. Liang, A. Mead, M. S. R. Siddiqui, L. Van Waerbeke, and A. Zhitnitsky, Phys. Rev. D 100, 043531 (2019).

[51] P. Gorham, Phys. Rev. D 86, 123005 (2012). 
[52] D. M. Jacobs, G. D. Starkman, and B. W. Lynn, Mon. Not. R. Astron. Soc. 450, 3418 (2015).

[53] E. T. Herrin, D. C. Rosenbaum, and V. L. Teplitz, Phys. Rev. D 73, 043511 (2006).

[54] D. H. Oaknin and A. R. Zhitnitsky, Phys. Rev. Lett. 94, 101301 (2005).

[55] A. Zhitnitsky, Phys. Rev. D 76, 103518 (2007).

[56] M. M. Forbes and A. R. Zhitnitsky, J. Cosmol. Astropart. Phys. 01 (2008) 023.

[57] K. Lawson and A. R. Zhitnitsky, J. Cosmol. Astropart. Phys. 01 (2008) 022.

[58] M. M. Forbes and A. R. Zhitnitsky, Phys. Rev. D 78, 083505 (2008).

[59] M. M. Forbes, K. Lawson, and A. R. Zhitnitsky, Phys. Rev. D 82, 083510 (2010).

[60] A. Zhitnitsky, Phys. Dark Universe 22, 1 (2018).

[61] X. Liang and A. Zhitnitsky, Phys. Rev. D 99, 023015 (2019).

[62] H. Fischer, X. Liang, Y. Semertzidis, A. Zhitnitsky, and K. Zioutas, Phys. Rev. D 98, 043013 (2018).

[63] X. Liang, A. Mead, M. S. R. Siddiqui, L. Van Waerbeke, and A. Zhitnitsky, Phys. Rev. D 101, 043512 (2020).

[64] P. Gorham and B. J. Rotter, Phys. Rev. D 95, 103002 (2017).

[65] K. Lawson and A. Zhitnitsky, Phys. Rev. D 95, 063521 (2017).

[66] M. G. Alford, A. Schmitt, K. Rajagopal, and T. Schfer, Rev. Mod. Phys. 80, 1455 (2008).

[67] K. Rajagopal and F. Wilczek, in At the Frontier of Particle Physics. Handbook of QCD, edited by M. Shifman and B. Ioffe (2000), Vol. 1-3, pp. 2061-2151.

[68] J. N. Bahcall, A. M. Serenelli, and S. Basu, Astrophys. J. 621, L85 (2005).

[69] C. Lunardini and O. L. G. Peres, J. Cosmol. Astropart. Phys. 08 (2008) 033.

[70] Y. Gando et al. (Super-Kamiokande Collaboration), Phys. Rev. Lett. 90, 171302 (2003).

[71] A. Gando et al. (KamLAND Collaboration), Astrophys. J. 745, 193 (2012).
[72] D. Barker, D. M. Mei, and C. Zhang, Phys. Rev. D 86, 054001 (2012).

[73] Q. Yue et al. (CDEX Collaboration), Phys. Rev. D 90, 091701 (2014).

[74] Z. Ahmed et al. (CDMS-II Collaboration), Phys. Rev. Lett. 106, 131302 (2011).

[75] Z. Ahmed et al. (CDMS-II Collaboration), arXiv:1203 .1309 .

[76] E. Armengaud et al. (EDELWEISS Collaboration), Phys. Rev. D 86, 051701 (2012).

[77] D. S. Akerib et al. (LUX Collaboration), Phys. Rev. Lett. 112, 091303 (2014).

[78] R. Agnese et al. (SuperCDMS Collaboration), Phys. Rev. Lett. 112, 241302 (2014).

[79] J. Angle et al. (XENON10 Collaboration), Phys. Rev. Lett. 107, 051301 (2011); 110, 249901(E) (2013).

[80] E. Aprile et al. (XENON100 Collaboration), Phys. Rev. Lett. 109, 181301 (2012).

[81] C. E. Aalseth et al. (CoGeNT Collaboration), arXiv:1401 .3295 .

[82] M. Tanabashi et al. (Particle Data Group), Phys. Rev. D 98, 030001 (2018).

[83] L. M. Capparelli, G. Cavoto, D. Mazzilli, and A. D. Polosa, Phys. Dark Universe 9-10, 24 (2015); 11, 79(E) (2016).

[84] Y. Hochberg, Y. Kahn, M. Lisanti, C. G. Tully, and K. M. Zurek, Phys. Lett. B 772, 239 (2017).

[85] G. Cavoto, F. Luchetta, and A. D. Polosa, Phys. Lett. B 776, 338 (2018).

[86] D. Budker, V. V. Flambaum, X. Liang, and A. Zhitnitsky, Phys. Rev. D 101, 043012 (2020).

[87] D. Budker, V. V. Flambaum, and A. Zhitnitsky, arXiv:2003.07363.

[88] P. Jaikumar, M. Prakash, and T. Schafer, Phys. Rev. D 66, 063003 (2002).

[89] S. Reddy, M. Sadzikowski, and M. Tachibana, Nucl. Phys. A714, 337 (2003).

[90] S. Reddy, M. Sadzikowski, and M. Tachibana, Phys. Rev. D 68, 053010 (2003). 